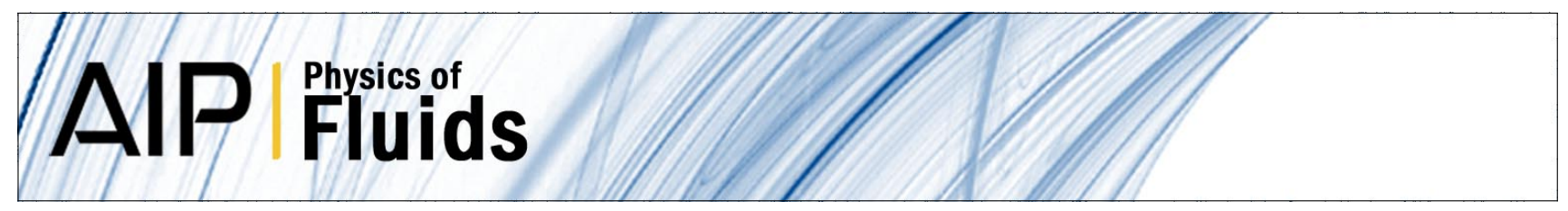

\title{
Non-invasive determination of external forces in vortex-pair-cylinder interactions
}

D. Hartmann, W. Schröder, and B. N. Shashikanth

Citation: Phys. Fluids 24, 061903 (2012); doi: 10.1063/1.4729613

View online: http://dx.doi.org/10.1063/1.4729613

View Table of Contents: http://pof.aip.org/resource/1/PHFLE6/v24/i6

Published by the American Institute of Physics.

\section{Related Articles}

Finite Rossby radius effects on vortex motion near a gap

Phys. Fluids 24, 066601 (2012)

Flow mediated interactions between two cylinders at finite Re numbers

Phys. Fluids 24, 043103 (2012)

The continuous spectrum of time-harmonic shear layers

Phys. Fluids 24, 034101 (2012)

The interaction of a vortex ring with a sloped sediment layer: Critical criteria for incipient grain motion

Phys. Fluids 24, 026604 (2012)

Numerical study of flow characteristics behind a stationary circular cylinder with a flapping plate

Phys. Fluids 23, 073601 (2011)

\section{Additional information on Phys. Fluids}

Journal Homepage: http://pof.aip.org/

Journal Information: http://pof.aip.org/about/about_the_journal

Top downloads: http://pof.aip.org/features/most_downloaded

Information for Authors: http://pof.aip.org/authors

\section{ADVERTISEMENT}

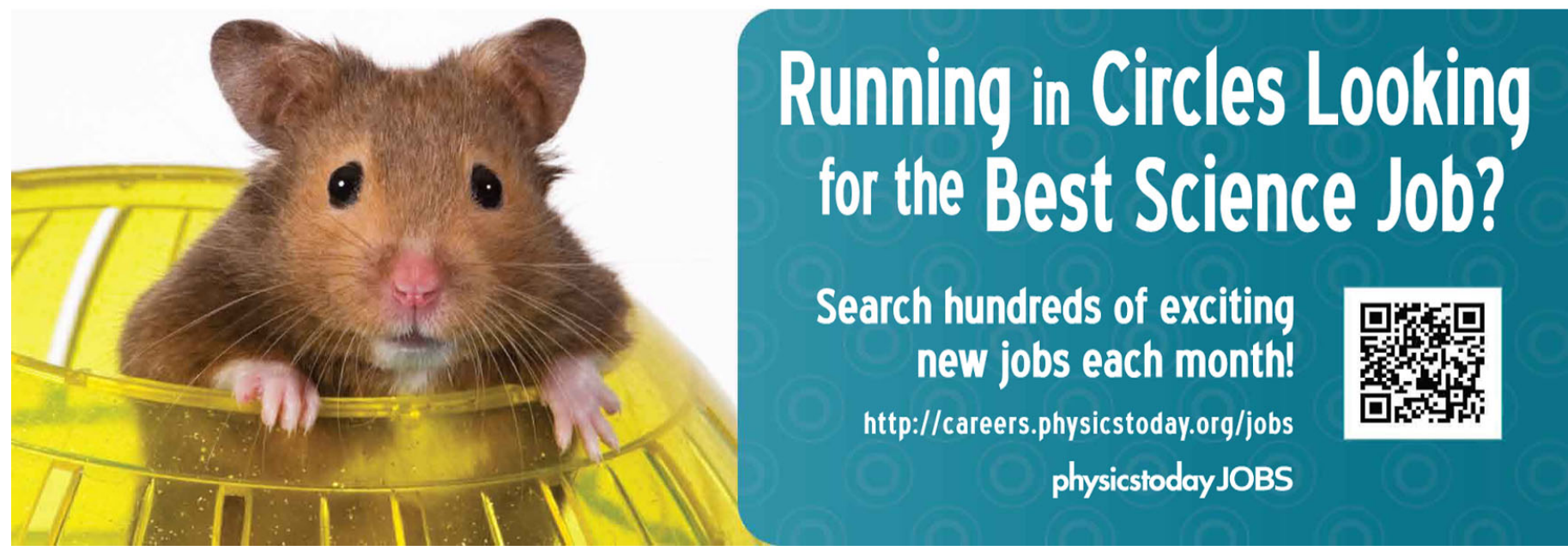




\title{
Non-invasive determination of external forces in vortex-pair-cylinder interactions
}

\author{
D. Hartmann, ${ }^{1, a)}$ W. Schröder, ${ }^{1, b)}$ and B. N. Shashikanth ${ }^{2, c)}$ \\ ${ }^{1}$ Institute of Aerodynamics, RWTH Aachen University, Wüllnerstr. 5a, \\ 52062 Aachen, Germany \\ ${ }^{2}$ Department of Mechanical and Aerospace Engineering, New Mexico State University, \\ Las Cruces, New Mexico 88003, USA
}

(Received 21 September 2011; accepted 24 April 2012; published online 25 June 2012)

Expressions for the conserved linear and angular momenta of a dynamically coupled fluid + solid system are derived. Based on the knowledge of the flow velocity field, these expressions allow the determination of the external forces exerted on a body moving in the fluid such as, e.g., swimming fish. The verification of the derived conserved quantities is done numerically. The interaction of a vortex pair with a circular cylinder in various configurations of motions representing a generic test case for a dynamically coupled fluid + solid system is investigated in a weakly compressible Navier-Stokes setting using a Cartesian cut-cell method, i.e., the moving circular cylinder is represented by cut cells on a moving mesh. The objectives of this study are twofold. The first objective is to show the robustness of the derived expressions for the conserved linear and angular momenta with respect to bounded and discrete data sets. The second objective is to study the coupled dynamics of the vortex pair and a neutrally buoyant cylinder free to move in response to the fluid stresses exerted on its surface. A comparison of the vortex-body interaction with the case of a fixed circular cylinder evidences significant differences in the vortex dynamics. When the cylinder is fixed strong secondary vorticity is generated resulting in a repeating process between the primary vortex pair and the cylinder. In the neutrally buoyant cylinder case, a stable structure consisting of the primary vortex pair and secondary vorticity shear layers stays attached to the moving cylinder. In addition to these fundamental cases, the vortex-pair-cylinder interaction is studied for locomotion at constant speed and locomotion at constant thrust. It is shown that a similar vortex structure like in the neutrally buoyant cylinder case is obtained when the cylinder moves away from the approaching vortex pair at a constant speed smaller than the vortex pair translational velocity. Finally, the idealized symmetric settings are complemented by an asymmetric interaction of a vortex pair and a cylinder. This case is discussed for a fixed and a neutrally buoyant cylinder to show the validity of the derived relations for multi-dimensional body dynamics. ( 2012 American Institute of Physics. [http://dx.doi.org/10.1063/1.4729613]

\section{INTRODUCTION}

A dynamically coupled fluid-structure system involving coherent vorticity is the most appropriate framework to study locomotion problems in nature, such as the swimming of fish, other neutrally buoyant marine animals, ${ }^{7,8,29}$ and related biologically inspired designs of small, autonomous underwater systems - an area of research that is now commonly referred to as biomimetic locomotion.

\footnotetext{
a) Current address: Department of Engineering and Applied Science, California Institute of Technology, California 91106, USA. Electronic mail: office@ aia.rwth-aachen.de.

b) Electronic mail: office@aia.rwth-aachen.de.

c) Electronic mail: shashi@nmsu.edu.
} 
From a systems perspective all these problems are "dynamics + control" problems, the resulting system trajectory being the result of control actuation applied to the body in response to the coupled dynamics. Adopting this perspective, it is a natural first step to study the dynamics without control. Unlike fluid-structure systems studied in other areas such as classical aircraft aerodynamics, Stokes flow, or vortex-induced vibration (VIV) dynamically coupled interaction problems present new challenges since the solid motion is no longer prescribed, the flow is unsteady, and possibly, aperiodic and no small-body, linearization, or quasi-steady assumptions are valid. Currently, there is growing interest in such problems not only from a theoretical ${ }^{6,9,34,35,40-43}$ but also from a numerical ${ }^{5,22}$ and an experimental viewpoint. ${ }^{2,46}$ When aquatic locomotion is considered, experiments with living animals are indispensable to understand their propulsive mechanisms. The wake flow of naturally swimming fish has been characterized using the technique of high-resolution, high-speed particleimage velocimetry in various studies. ${ }^{28,29,47}$ Nevertheless, it is still a great challenge to separate the external forces, i.e., thrust, and the fluid forces, i.e., drag, to determine the swimming efficiency of fish from these flow measurements. ${ }^{39,46}$

It is fair to conclude that regarding the propulsion system much remains to be done. To improve the knowledge a step-by-step approach is to be pursued. That is, first the analysis should be performed for generic configurations before the geometric complexity of, say, real configurations such as swimming fish is added to the problem. It is interesting to note that such a fundamental study using a body of simple geometry to elucidate basic principles of vortex-body interactions is still missing. Indeed, if we compare with a standard textbook — and the well-researched-topic on vortex shedding behind a stationary circular cylinder in a uniform stream, the case when the cylinder is free to move and interacts with a vortical flow field has hardly been investigated. Given the variety of flow scenarios encountered in swimming and locomotion problems, the choice of a canonical problem to study is not obvious. For example, a circular cylinder could be given an initial velocity and the ensuing coupled dynamics of the vortex wake and the cylinder motion be studied. Alternatively, an ambient uniform flow could be turned on to set the cylinder in motion. The model problem focused on in this study is largely motivated by the previous theoretical work and experimental work cited above-in particular, the symmetric point vortex configurations considered by Shashikanth ${ }^{41}$ and the vortex ring-sphere experiments of Allen $e t$ al. ${ }^{2} \mathrm{~A}$ symmetric viscous vortex pair interacts with a neutrally buoyant circular cylinder, which is free to move in response to the fluid force. A detailed comparison is made with the flow fields of a fixed cylinder and a cylinder translating at a constant speed. Furthermore, the case in which a constant external force accelerates the cylinder towards the approaching vortex pair and a fully multi-dimensional case, in which the vortex pair asymmetrically interacts with the cylinder, are analyzed. The central part of this study is the relation of the observed vortex dynamics due to the motion of the neutrally buoyant cylinder and the force on the fixed cylinder making use of linear and angular momentum conservation laws for the combined fluid + solid system. The derived expressions show the relation of the forces and the fluid + solid dynamics based on the bounded and discrete flow velocity field computed in the numerical simulations.

The paper is organized as follows. In Sec. II, the governing equations of our model are presented, before a brief description of the numerical method to solve these equations and results for a validation test case are provided in Sec. III. The linear and angular momentum conservation laws for the combined fluid + solid system are derived in Sec. IV. Simulations and data for vortex-cylinder interactions for different cases are presented and discussed in Sec. V. Finally, the findings of this study are summarized in Sec. VI.

\section{GOVERNING EQUATIONS}

\section{A. Equations governing compressible flow}

We study the interaction of a viscous fluid with a rigid solid body, which accelerates and rotates in response to the force and moment of force exerted by the fluid. The problem can be fully described by rigid body equations coupled to the non-dimensional Navier-Stokes equations for 
compressible flows ${ }^{3}$

$$
\frac{\partial \mathbf{Q}}{\partial t}+\nabla \cdot \overline{\mathbf{H}}=\mathbf{0},
$$

where $\mathbf{Q}=[\varrho, \varrho \mathbf{u}, \varrho E]^{T}$ denotes the vector of the conservative variables and the quantities $\varrho, \mathbf{u}$ $E=e+\frac{\mathbf{u}^{2}}{2}$, and $e$ denote the density, the velocity vector, the specific total energy, and the specific internal energy, respectively. The flux vector can be written

$$
\overline{\mathbf{H}}=\left(\begin{array}{c}
\varrho \mathbf{u} \\
\varrho \mathbf{u}+p \\
\mathbf{u}(\varrho E+p)
\end{array}\right)+\frac{1}{\operatorname{Re}}\left(\begin{array}{c}
0 \\
\overline{\boldsymbol{\tau}} \\
\overline{\boldsymbol{\tau}} \cdot \mathbf{u}+\mathbf{q}
\end{array}\right)
$$

using the Reynolds number $R e=\frac{\varrho_{\infty} a_{0} l}{\mu_{\infty}}$ based on the speed of sound at rest $a_{0}=\sqrt{\gamma p_{0} / \varrho_{0}}$ and the characteristic length $l$. The quantities $p, \gamma$, and $\overline{\boldsymbol{\tau}}$ denote the pressure, the ratio of specific heats, and the second-rank stress tensor, which can be formulated

$$
\overline{\boldsymbol{\tau}}=-2 \mu \overline{\mathbf{S}}+\frac{2}{3} \mu(\boldsymbol{\nabla} \cdot \mathbf{u}) \overline{\mathbf{I}}
$$

assuming a Newtonian fluid and zero bulk viscosity. The dynamic viscosity $\mu$ is computed using Sutherland's law

$$
\mu=\mu_{\infty}\left(\frac{T}{T_{\infty}}\right) \frac{T_{\infty}+S}{T+S}
$$

where $T$ is the static temperature and the constant $S$ is set $S=111 \mathrm{~K}$, which is valid at moderate temperatures. In Eq. (3), the quantities $\overline{\mathbf{I}}$ and $\overline{\mathbf{S}}$ are the unit tensor and the rate-of-strain tensor

$$
\overline{\mathbf{S}}=\frac{1}{2}\left(\nabla \mathbf{u}+(\nabla \mathbf{u})^{T}\right),
$$

respectively. The vector of heat conduction $\mathbf{q}$ is expressed by Fourier's law

$$
\mathbf{q}=-\frac{k}{\operatorname{Pr}(\gamma-1)} \nabla T .
$$

The Prandtl number $\operatorname{Pr}=\frac{\mu_{\infty} c_{p}}{k_{\infty}}$ contains the specific heat at constant pressure $c_{p}$. Finally, the thermal conductivity is evaluated from $k(T) \propto \mu(T)$, which holds for a constant Prandtl number.

Using the Gauss divergence theorem, the integral form of the Navier-Stokes equations is obtained as

$$
\int_{V} \frac{\partial \mathbf{Q}}{\partial t} d V+\int_{A} \overline{\mathbf{H}} \cdot \mathbf{n} d A=\mathbf{0},
$$

where $\mathbf{n}$ is the outward unit normal vector on the surface $d A$.

\section{B. Equations governing the motion of rigid bodies}

For a cylinder free to move under the influence of the fluid forces, Newton's law of motion can be formulated in non-dimensional form ${ }^{11}$

$$
\frac{d^{2} \mathbf{X}}{d t^{2}}=\frac{\mathbf{C}}{2 m_{\text {red }}}
$$

where $\mathbf{X}$ denotes the position vector of the structure center of mass in a spatially fixed frame. The force coefficient vector $\mathbf{C}$ and the reduced mass $m_{\text {red }}$ are defined

$$
\begin{aligned}
\mathbf{C} & =\frac{2 \mathbf{F}}{\varrho u_{\infty}^{2} D}, \\
m_{r e d} & =\frac{m_{B}}{\varrho D^{2}},
\end{aligned}
$$


where $D, \mathbf{F}, m_{B}$, and $u_{\infty}$ denote the reference length, the force acting on the body, the mass of the body, and the freestream velocity of the fluid, respectively.

The angular velocity $\boldsymbol{\Omega}$ of the structure is governed by

$$
\overline{\mathbf{J}}_{r e d} \cdot \frac{d \boldsymbol{\Omega}}{d t}=\frac{1}{2} \mathbf{C}_{M, \mathbf{X}}
$$

where the reduced moment of force $\mathbf{C}_{M, \mathbf{X}}$ and the reduced moment of inertia $\overline{\mathbf{J}}_{\text {red }}$ about the structure center of mass $\mathbf{X}$ are defined

$$
\begin{aligned}
\mathbf{C}_{M, \mathbf{X}} & =\frac{2 \mathbf{M}_{\mathbf{X}}}{\varrho u_{\infty}^{2} D^{2}}, \\
\overline{\mathbf{J}}_{r e d} & =\frac{\overline{\mathbf{J}}}{\varrho D^{4}} .
\end{aligned}
$$

The quantities $\mathbf{M}_{\mathbf{X}}$ and $\overline{\mathbf{J}}$ denote the moment of force vector about the structure center of mass and the moment of inertia tensor, respectively.

\section{Fluid-structure coupling}

The most widely used strategy to couple the fluid and structure equations is the so-called partitioned approach, ${ }^{1,4,10}$ in which these equations are solved separately and coupled by matching boundary conditions at the fluid-structure interface $\Gamma$. As a boundary condition for the fluid, the no-slip condition prescribed on the structure surface $\Gamma$ of the non-deformable body implies

$$
\left.\mathbf{u}\right|^{\Gamma}=\mathbf{U}+\sum_{j} \frac{\Omega_{j}}{2 \pi r_{j}}\left(\mathbf{n} \times \mathbf{e}_{j}\right)
$$

where $\mathbf{U}=d \mathbf{X} / d t$ and $\mathbf{e}_{j}$ denote the velocity of the center of mass (centroid) of the body in a spatially fixed frame and the unit vector along the principal axis $j$ of the Cartesian coordinate system passing through the centroid, respectively, and $r_{j}$ denotes the normal distance from this axis. Hence, the fluid velocity at the fluid-structure interface is imposed by the translational motion of the structure and its rotation. This motion results from the acceleration by the fluid force $\mathbf{F}$

$$
\mathbf{F}=\int_{\Gamma}\left(-p \mathbf{n}+\frac{1}{\operatorname{Re}} \overline{\boldsymbol{\tau}} \cdot \mathbf{n}\right) d \mathbf{x}^{2},
$$

where $\mathbf{n}$ is the outward normal vector on $\Gamma$ and $\Gamma$ is assumed to be a surface in three-dimensional space. Reducing Eq. (10) to the two-dimensional case, where $\Gamma$ represents a curve, is straightforward. Similarly, the rotation results from the moment which is exerted on the structure by the fluid and is computed in vector form by

$$
\mathbf{M}=\int_{\Gamma} \frac{1}{\operatorname{Re}}(\overline{\boldsymbol{\tau}} \cdot \mathbf{n}) \times \mathbf{n} d \mathbf{x}^{2}
$$

\section{NUMERICAL METHOD}

\section{A. Discretization of the Navier-Stokes equations}

The Navier-Stokes equations (1) are iteratively integrated in time using a 5-stage second-order accurate Runge-Kutta scheme optimized for stability using a globally computed time step. To spatially discretize the equations a cut-cell finite-volume formulation on a Cartesian non-boundaryfitted grid is used at globally second-order accuracy. In this method, the inviscid flux is formulated based on a modified version of the advection upstream splitting method (AUSM) originally proposed by Liou and Steffen. ${ }^{26}$ The required variables on the surface are reconstructed at secondorder accuracy using a monotone upstream centered scheme for conservation laws introduced by van Leer $^{24}$ based on a linear least-squares estimate of the cell center gradients. As the computations 
are performed in a body-fixed frame of reference attached to the cylinder the body velocity $U_{j}$ in the $j$ direction appears in the convective transport term and is included in the Mach number expressed as $M_{j}=\left(u_{j}+U_{j}\right) / a$. The viscous flux is computed using a distance-weighted convex combination of the cell-center gradients, which reduces to a centered scheme in uniform mesh areas.

The cut cells at the embedded boundaries are reshaped such that these boundaries are cut out of the computational domain as illustrated in Figure 3(a). In the final grid, the embedded boundaries are represented by the piecewise linear boundary surfaces, one of which is assigned to each boundary cell. Cut cells of small cell volume, which are commonly referred to as small cells, are treated using a cell-merging/cell-linking procedure. Furthermore, a ghost cell is introduced for each boundary cell and connected with it through the boundary surface, i.e., the boundary surface flux is computed based on the state variables and their gradients in the boundary cell and the ghost cell. The ghost cell variables are updated using the prescribed boundary conditions, i.e., Dirichlet boundary conditions for the velocities and homogeneous Neumann boundary conditions for the pressure and the density. Expressions for the derivatives of the velocity vector required to compute the stress tensor $\overline{\boldsymbol{\tau}}$, Eq. (3), on the boundary surfaces of cut cells can be derived using a transformation into a locally body-fitted coordinate system. For details on the overall numerical method and a validation of the flow solver for several two-dimensional and three-dimensional flows with fixed embedded boundaries the reader is referred to Hartmann et al. ${ }^{18}$

\section{B. Solution of the structure equations}

The motion of the center of mass $\mathbf{X}(t)$ is computed by solving Eq. (7). A central difference scheme with lagged approximation for the first derivative is used following Ahn and Kallinderis, ${ }^{1}$ which yields for the second derivative in Eq. (7)

$$
\left.\frac{\delta^{2} \mathbf{X}}{\delta t^{2}}\right|^{w}=\frac{1}{\Delta t^{2}}\left(\mathbf{X}^{w+1}-2 \mathbf{X}^{w}+\mathbf{X}^{w-1}\right)
$$

and for the first derivative

$$
\left.\frac{\delta \mathbf{X}}{\delta t}\right|^{w}=\frac{1}{\Delta t}\left(\mathbf{X}^{w}-\mathbf{X}^{w-1}\right),
$$

where $\delta$ denotes a discrete derivative. Using Eqs. (12) and(13), the position vector $\mathbf{X}^{w+1}$ is computed by

$$
\mathbf{X}^{w+1}=\mathbf{X}^{w}+\Delta t^{2} \frac{\mathbf{C}^{w}}{m_{\text {red }}}+\left.\Delta t \frac{\delta \mathbf{X}}{\delta t}\right|^{w},
$$

and the velocity of the structure $\partial \mathbf{X}^{w+1} / \partial t$ required in Eq. (9) is obtained from Eq. (13).

Analogously, the angular velocity $\boldsymbol{\Omega}^{w+1}$ is computed from Eq. (8) by

$$
\boldsymbol{\Omega}^{w+1}=\boldsymbol{\Omega}^{w}+\frac{\Delta t}{2} \overline{\mathbf{J}}_{r e d}^{-1} \cdot \mathbf{C}_{M, \mathbf{X}}^{w}
$$

\section{Validation}

The numerical method was validated for a variety of cold flow problems involving curved embedded boundaries in Hartmann et al. ${ }^{12,13,18}$ as well as for reacting flows in Hartmann et al. ${ }^{14,15,17}$ To validate the numerical method for the fluid-structure interaction problem, we performed simulations of vortex induced vibrations of a circular cylinder. In these simulations, a sponge layer formulation for the pressure and the density as presented in Hartmann et al. ${ }^{12}$ is used to drive the solution variables on the far-field boundaries towards the freestream values as well as to avoid spurious waves in the vicinity of these boundaries. The Mach number $M$ based on the reference velocity is chosen to be $M=0.1$ such that the results are comparable to simulations for incompressible flows.

We consider the flow past an elastically mounted circular cylinder with diameter $D$ in uniform freestream restrained to move in the cross-flow direction. We adopt the commonly made assumptions 


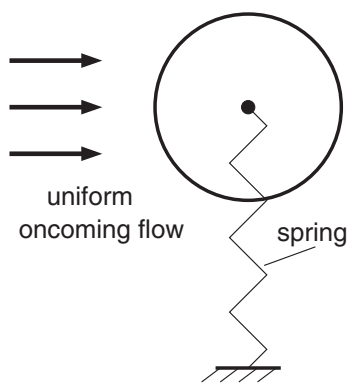

(a)

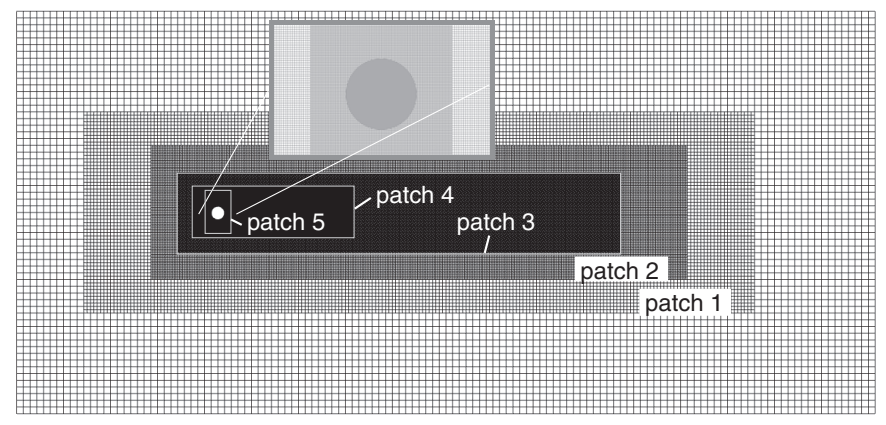

(b)

FIG. 1. VIV of a circular cylinder: (a) sketch of the test case set-up and (b) computational grid used for the simulations. Mesh refinement patches around the cylinder are highlighted by blue boxes for better visibility. The coarser grids are obtained by removing mesh refinement patches from the illustrated reference grid, i.e., removing patch 5 from the reference grid yields the grid $\mathrm{C} 1$ and removing patch 4 from $\mathrm{C} 1$ yields the grid $\mathrm{C} 2$, respectively. The inset shows a close-up view of the grid near the circular cylinder, where all boundary cells are shown as cut cells such that the representation of the cylinder on the non-boundary-fitted grid becomes visible.

that the spring mounting does not directly influence the flow and inhibits a rotation of the cylinder. This essentially reduces the problem to a standard cylinder flow problem, where the cylinder is allowed to transversely move in response to the fluid force and the force exerted by the elastic spring mounting. The set-up is shown schematically in Figure 1(a). The Reynolds number based on the freestream velocity of the flow and the cylinder diameter is $R e_{D}=150$ and the flow is assumed laminar and two-dimensional. For this configuration, the cylinder is known to couple with the unsteady vortex shedding process when the vortex formation frequency comes close to the natural vibration frequency of the cylinder-spring system, see Williamson and Govardhan ${ }^{49}$ for a review. This coupling results in a vibration of the cylinder with an amplitude larger than half the cylinder diameter, which occurs in response to the unsteady surface pressure force due to the vortex shedding.

For the simulations, we use a computational domain $\mathcal{D}:[-15 D, 49 D] \times[-15 D, 15 D]$. At the beginning of the computations corresponding to time level $t=0$, the cylinder is materialized with its center of mass slightly offset from the reference position. This initially generates a spring force which in turn initiates the oscillatory motion of the cylinder. To be more precise, the initial position of the cylinder center is prescribed to be $(X, Y)=(0,-0.05 D)$ and its reduced mass is taken to be $m_{\text {red }}=2$. The computational grid, which is shown in Figure 1(b), is clustered along the expected path of the cylinder and in the near cylinder wake regions using local mesh refinement. For the reduced velocity, we consider a parameter space $U_{\text {red }}=3, \ldots, 7$, which allows a direct comparison with the simulation results of Ahn and Kallinderis ${ }^{1}$ and Borazjani et al. ${ }^{4}$

First, we perform a grid refinement study based on four different grids. The reference grid, which is composed of all the mesh patches shown in Figure 1(b), possesses a mesh spacing of $\Delta x \approx 0.016 D$ around the circular cylinder. The coarser grids $\mathrm{C} 1$ and $\mathrm{C} 2$ are obtained by successively removing the mesh refinement patches. In addition to these grids, a finer grid is also investigated. On this grid, another refinement patch is laid into patch 5. The results obtained for the parameter $U_{\text {red }}=4$ are shown in Figure 2(a), where the transverse position $Y$ of the cylinder mass center is plotted vs time. The data clearly show that the solutions converge on successively finer grids. The plots corresponding to the fine grid and reference grid solutions match, suggesting that grid converged solutions are obtained on the reference grid, which is used hereafter. As a quantitative measure of the degree of uncertainty for the results on the reference grid we evaluate the accumulated error in phase and the error in amplitude of the cylinder motion computed on this grid relative to the data obtained on the fine grid. More specifically, the errors are assessed for the last top dead center position of the cylinder plotted in Figure 2(a), which is computed to be reached at time level $t=57.29$ at an amplitude of $\Delta Y=0.565$ on the fine grid. The relative errors in phase and amplitude for the reference grid results are $5.81 \times 10^{-2} \mathrm{rad}$ and $-2.42 \times 10^{-3}$, respectively. As a comparison, the corresponding errors 


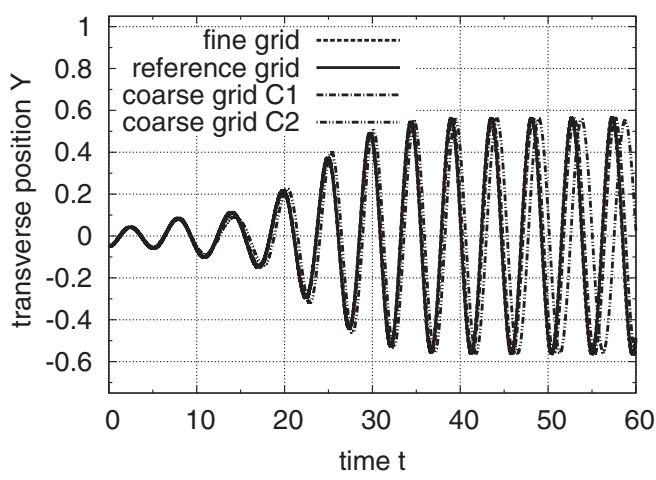

(a)

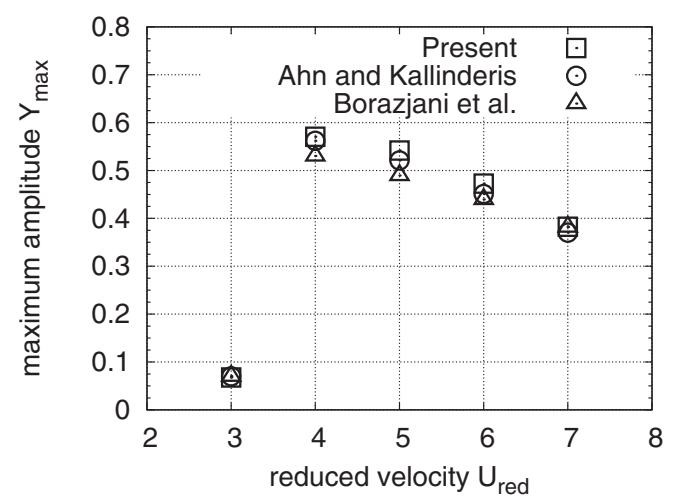

(b)

FIG. 2. VIV of a circular cylinder at $R e_{D}=150$ and $m_{\text {red }}=2$ : (a) transverse position $Y$ of the body mass center vs time on different grids; note that the reference grid and the fine grid solutions perfectly match and (b) maximum amplitude $Y_{\max }$ of the body mass center as a function of the spring stiffness.

in phase and amplitude obtained on the coarse grid C2 are $2.02 \times 10^{0} \mathrm{rad}$ and $-2.61 \times 10^{-2}$, respectively.

Next, we discuss the results obtained for different values of the parameter $U_{\text {red }}$ on the reference grid. The data are summarized and compared with published results in Figure 2(b) evidencing the present results to be in convincing agreement with the data from the literature. As for the maximum amplitude $Y_{\max }$ of the oscillatory cylinder motion, the greatest spread in the data is found for the parameter $U_{\text {red }}=5$, at which Ahn and Kallinderis ${ }^{1}$ and Borazjani et al. ${ }^{4}$ report values of $Y_{\max }=0.52$ and $Y_{\max }=0.49$, respectively, while the present computations yield $Y_{\max }=0.54$. As illustrated in Figure 2(b), the maximum vortex induced vibration amplitude of the cylinder is reached for $U_{\text {red }}=4.0$, whereas for a stiffer structure at $U_{\text {red }}=3$ the vibration amplitude drops to very small values below $0.1 D$. For $U_{\text {red }}>5.0$ the vibration amplitude diminishes with increasing flexibility of the structure, i.e., with increasing values for the parameter $U_{\text {red }}$.

\section{MOMENTUM LAWS FOR THE FLUID + SOLID SYSTEM}

In this section, the momentum laws for the combined fluid + solid system are presented. There are many papers that derive force and moment expressions for bodies in a viscous flow. Some representative works are by $\mathrm{Wu},{ }^{50}$ Quartapelle et al.,${ }^{33} \mathrm{Wu}$ and $\mathrm{Wu},{ }^{51}$ and Howe $;{ }^{20}$ see also the texts by Saffman ${ }^{36}$ and Howe. ${ }^{21}$ For potential flows with isolated singularities, force and moment theories go back to Blasius and Lagally. ${ }^{27}$ However, momentum laws for dynamically coupled, or neutrally buoyant cylinder, cases, have received lesser attention. For inviscid models with singular distributions of vorticity, derivations can be found in Shashikanth et al. ${ }^{42,43}$ In principle, momentum laws for dynamically coupled systems can be inferred from the force and moment expressions in the references cited above but to make the paper self-contained the main steps of such a derivation from first principles are presented in the first subsection. Moreover, though all the simulations presented are for flows in $2 \mathrm{D}$, for the sake of generality the momentum laws are derived in both 3D and 2D. The key features are: expressing the momentum of the fluid in terms of moments of vorticity-a feature also invoked in the papers cited above-and imposing the no-slip boundary condition to simplify the integrals on the solid-fluid boundary.

Let the domain occupied by the body of mass $m_{B}$, volume $V_{B}$, and density $\varrho_{B}$ be denoted by $B, B \subset \mathbb{R}^{3}$, as illustrated in Figure 3(b) and its boundary be denoted by $\partial B$. Furthermore, let $D$ and $\widetilde{D}$ designate the externally unbounded domain occupied by the fluid and a bounded subset of $D$ with $\partial \widetilde{D}=\partial B \cup S$, respectively, where $S$ is a closed two-dimensional surface enclosing the body and most of the vorticity—see Main assumption to follow. The domain $\widetilde{D}$ may represent a computational domain in a numerical simulation or a measurement volume in an experiment. The 


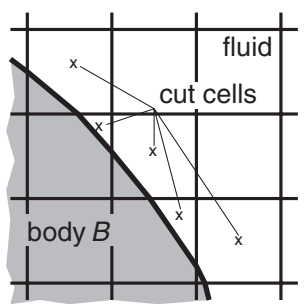

(a)

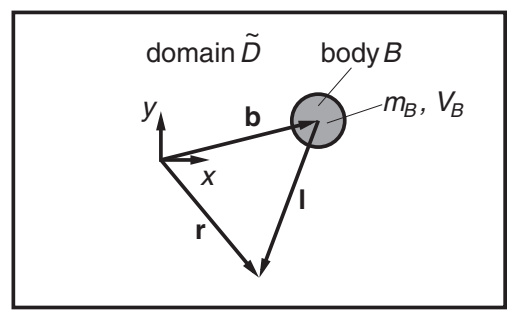

(b)

FIG. 3. Diagrams illustrating (a) Cartesian cut cells near the boundary and (b) the notation.

vector $\mathbf{n}_{i}$ denotes the inward pointing unit normal field on $\partial \widetilde{D}$ in a spatially fixed frame. The vector quantities $\mathbf{U}$ and $\boldsymbol{\Omega}$ and the tensor $\overline{\mathbf{J}}$ denote the velocity of the center of mass and the angular velocity of the body in a spatially fixed frame as well as the moment of inertia of the body referred to principal axes passing through the center of mass. Finally, let $\mathbf{I}$ and $\mathbf{b}$, respectively be the position vector from and of the body center of mass (centroid) in a spatially fixed frame, as illustrated in Figure 3(b).

Far-field decay rates in $\mathbb{R}^{3}$. Since the fluid domain is unbounded away from the body, a brief discussion of velocity decay rates is in order. Recall ${ }^{3}$ that in a flow with no boundaries, the far-field velocity field can be described by $\mathbf{u}=O\left(1 /|\mathbf{x}|^{3}\right)$ provided $\int_{\mathbb{R}^{3}} \omega d V=0$. In turn, the latter holds if the far-field vorticity field satisfies $\omega=O\left(1 /|\mathbf{x}|^{4}\right)$ (using integration by parts). In this problem, the fluid has an inner boundary, coincident with the boundary of the moving body. In general, $\int_{\tilde{D}} \boldsymbol{\omega} d V \equiv-\int_{\partial \tilde{D}} \mathbf{x} \boldsymbol{\omega} \cdot \mathbf{n}_{i} d A$ will not be zero, even as $\tilde{D} \rightarrow D$, because of the presence of the body boundary integral. However, because of the no-slip boundary condition the expression $\boldsymbol{\omega} \cdot \mathbf{n}_{i}$ is continuous at the body-fluid boundary. It follows that the integral

$$
\int_{\tilde{D} \cup B} \omega d V:=\int_{\tilde{D}} \omega d V+\int_{B} \omega d V=\int_{\tilde{D}} \omega d V+\int_{B} 2 \Omega d V,
$$

where the vorticity field of the body is the curl of the body velocity field, is well-defined and is equal to $-\int_{S} \mathbf{x} \boldsymbol{\omega} \cdot \mathbf{n}_{i} d A$. And so if $\boldsymbol{\omega}=O\left(1 /|\mathbf{x}|^{4}\right)$ (at least) then $\int_{D \cup B} \omega d V=0$. To obtain the corresponding decay rate of the velocity field, the definition of vorticity $\omega=\nabla \times \mathbf{u}$ can be used to show that $\mathbf{u}=O\left(1 /|\mathbf{x}|^{3}\right)$. The far-field behavior for the fluid + body system in $\mathbb{R}^{3}$ is thus summarized as follows:

$$
\omega=O\left(1 /|\mathbf{x}|^{4}\right) \Rightarrow \int_{D \cup B} \omega d V=0 \text { and } \mathbf{u}=O\left(1 /|\mathbf{x}|^{3}\right) .
$$

For faster $\omega$ decay rates the integral continues to be zero and the decay rate of $\mathbf{u}$ is also faster.

\section{A. 3D neutrally buoyant cylinder}

Main assumption. We will assume that the vorticity $\omega$ decays sufficiently rapidly at large distances so that at any time instant $t$ there exists an $S$ such that on and outside $S$ the velocity field is effectively irrotational and Bernoulli's equation is applicable. Note that this assumption also implies that no vorticity is created by the action of viscous stresses in this far-field region. Newton's Second Law for linear and angular momentum applied to the control volume $B \cup \widetilde{D}$, with $\varrho_{B}=\varrho$ and $r:=|\mathbf{x}|$, implies

$$
\begin{gathered}
V_{B} \frac{d \mathbf{U}}{d t}+\frac{d}{d t}\left(\frac{1}{2}\left\{\int_{\widetilde{D}}(\mathbf{x} \times \boldsymbol{\omega}) d V+\int_{\partial B} \mathbf{x} \times\left(\mathbf{n}_{i} \times \mathbf{u}\right) d A\right\}\right)=\mathbf{F}_{S}, \\
\frac{d}{d t}\left(V_{B} \mathbf{b} \times \mathbf{U}+\overline{\mathbf{J}} \cdot \Omega\right)-\frac{1}{2} \frac{d}{d t}\left(\int_{\widetilde{D}}\left(r^{2} \boldsymbol{\omega}\right) d V+\int_{\partial B} r^{2}\left(\mathbf{n}_{i} \times \mathbf{u}\right) d A\right)=\mathbf{M}_{S},
\end{gathered}
$$


where

$$
\begin{aligned}
& \mathbf{F}_{S}=\int_{S} \mathbf{u}\left(\mathbf{u} \cdot \mathbf{n}_{i}\right) d A-\frac{d}{d t}\left(\frac{1}{2} \int_{S} \mathbf{x} \times\left(\mathbf{n}_{i} \times \mathbf{u}\right) d A\right)+\frac{1}{\varrho} \int_{S}(p \overline{\mathbf{I}}-\overline{\mathbf{T}}) \cdot \mathbf{n}_{i} d A, \\
& \mathbf{M}_{S}=\int_{S} \mathbf{x} \times \mathbf{u}\left(\mathbf{u} \cdot \mathbf{n}_{i}\right) d A+\frac{1}{2} \frac{d}{d t} \int_{S} r^{2}\left(\mathbf{n}_{i} \times \mathbf{u}\right) d A+\frac{1}{\varrho} \int_{S}\left(\mathbf{x} \times\left((p \overline{\mathbf{I}}-\overline{\mathbf{T}}) \cdot \mathbf{n}_{i}\right)\right) d A
\end{aligned}
$$

(note that $\mathbf{n}_{i}$ is the unit inward normal on $S$ ). In the above, the linear and angular momentum integrals of the fluid in $\tilde{D}$ have been transformed using the vector identities

$$
\begin{aligned}
\int_{\tilde{D}} \mathbf{u} d A & =\frac{1}{2} \int_{\tilde{D}} \mathbf{x} \times(\nabla \times \mathbf{u}) d V+\frac{1}{2} \int_{\partial \tilde{D}} \mathbf{x} \times\left(\mathbf{n}_{i} \times \mathbf{u}\right) d A, \\
\int_{\tilde{D}} \mathbf{x} \times \mathbf{u} d A & =-\frac{1}{2} \int_{\tilde{D}} r^{2}(\nabla \times \mathbf{u}) d V-\frac{1}{2} \int_{\partial \tilde{D}} r^{2}\left(\mathbf{n}_{i} \times \mathbf{u}\right) d A .
\end{aligned}
$$

From the Main assumption, it follows that $\mathbf{u}=\nabla \phi$ on $S$ where $\phi$ is the velocity potential function defined in a local neighborhood of $S$. This, combined with Bernoulli's equation on $S$, can be used to show that the terms $\mathbf{F}_{S}$ and $\mathbf{M}_{S}$-minus the shear stress integral-go to zero as $S \rightarrow \infty$, as in Shashikanth et al. ${ }^{43}$ The shear stress integral terms can be expressed as

$$
-v \int_{S} \nabla \mathbf{u} \cdot \mathbf{n}_{i} d A \text { and }-v \int_{S}\left(\mathbf{x} \times(\nabla \mathbf{u}) \cdot \mathbf{n}_{i}\right) d A .
$$

From the Main assumption and the decay rates of the fields in $\mathbb{R}^{3}$, it follows that both these terms go to zero as $S \rightarrow \infty$.

And so

$$
\begin{aligned}
& \frac{d \mathbf{L}}{d t}=0, \\
& \frac{d \mathbf{A}}{d t}=0,
\end{aligned}
$$

where

$$
\begin{aligned}
& \mathbf{L}=V_{B} \mathbf{U}+\left(\frac{1}{2}\left(\int_{D}(\mathbf{x} \times \boldsymbol{\omega}) d V+\int_{\partial B} \mathbf{x} \times\left(\mathbf{n}_{i} \times(\mathbf{U}+\Omega \times \mathbf{l})\right) d A\right)\right), \\
& \mathbf{A}=V_{B} \mathbf{b} \times \mathbf{U}+\overline{\mathbf{J}} \cdot \Omega-\frac{1}{2}\left(\int_{D}\left(r^{2} \boldsymbol{\omega}\right) d V+\int_{\partial B} r^{2}\left(\mathbf{n}_{i} \times(\mathbf{U}+\Omega \times \mathbf{I})\right) d A\right) .
\end{aligned}
$$

As illustrated in Figure 3(a), now express the position vector as

$$
\mathbf{x}=\mathbf{b}+\mathbf{l},
$$

and make use of the following: the no-slip boundary condition, $\int_{B} \mathbf{l} d V=0$, Stokes theorem and integration by parts. It can be shown-after some algebra-that

$$
\begin{aligned}
& \mathbf{L}=\mathbf{b} \times\left(\Omega V_{B}+\frac{1}{2} \int_{D} \omega d V\right)+\frac{1}{2} \int_{D} \mathbf{l} \times \omega d V \\
& \mathbf{A}=-b^{2}\left(\Omega V_{B}+\frac{1}{2} \int_{D} \omega d V\right)-\frac{1}{2} \int_{D} l^{2} \omega d V-\int_{B} l^{2} \Omega d V-\int_{D} \mathbf{1} \cdot \mathbf{b} \omega d V .
\end{aligned}
$$

The terms within parenthesis are nothing but $(1 / 2) \int_{D \cup B} \omega d V$. From the Main assumption and the decay rates of the fields in $\mathbb{R}^{3}$, it follows that

$$
\mathbf{L}=\frac{1}{2} \int_{D} \mathbf{l} \times \omega d V
$$




$$
\mathbf{A}=-\frac{1}{2} \int_{D} l^{2} \boldsymbol{\omega} d V-\int_{B} l^{2} \Omega d V-\int_{D} \mathbf{l} \cdot \mathbf{b} \boldsymbol{\omega} d V,
$$

and both these quantities are conserved by the dynamics.

It is clear from Eqs. (26) and (27) that the full three-dimensional vorticity field is required to compute the conserved quantities and that planar velocity field data are insufficient. It goes without saying that this is quite demanding in terms of the anticipated use of these relations in experimental analyses. Nevertheless, modern experimental techniques such as the holographic particle image velocity have been shown to be mature enough to record three-dimensional flow velocity fields even in complex configurations. ${ }^{37,38,45}$ In quasi two-dimensional cases the relations (26) and (27) simplify such that only the planar velocity field, which can be obtained using standard experimental measurement techniques, is required. These relations are derived next.

\section{B. 2D neutrally buoyant cylinder}

The far-field terms minus the shear stress integrals (18) can be shown to go to zero as in the $3 \mathrm{D}$ case (see also Shashikanth et al. ${ }^{42}$ ). In $2 \mathrm{D}, \int_{D \cup B} \omega d A:=\int_{D} \omega d A+2 \Omega A_{B}$ can be non-zero and the far-field velocity behaves as (a) $\mathbf{u}=O(1 /|\mathbf{x}|)$ if $\int_{D} \omega d A+2 \Omega A_{B} \neq 0$ and (b) $\mathbf{u}=O\left(1 /|\mathbf{x}|^{2}\right)$ if $\int_{D} \omega d A+2 \Omega A_{B}=0$. The first of the two shear stress integral terms (18) vanishes but, for (a), the second may not. And so, conservation of $\mathbf{L}$ continues to hold but conservation of $\mathbf{A}$ may not hold. For flows without boundaries this is a result that goes back to Poincaré ${ }^{32}$ and is also discussed in, for example, Saffman. ${ }^{36}$

In $2 \mathrm{D}, \mathbf{A}$ has the same form as above (with $\overline{\mathbf{J}}$ reduced to a scalar) and $\mathbf{L}$ takes the form

$$
\mathbf{L}=A_{B} \mathbf{U}+\left\{\int_{D}(\mathbf{x} \times \boldsymbol{\omega}) d A+\oint_{\partial B} \mathbf{x} \times\left(\mathbf{n}_{i} \times(\mathbf{U}+\Omega \times \mathbf{l})\right) d s\right),
$$

i.e., the factor $1 / 2$ is no longer present. Transforming using $\mathbf{b}$ and $\mathbf{l}$

$$
\begin{aligned}
& \mathbf{L}=\mathbf{b} \times\left(2 \Omega A_{B}+\int_{D} \boldsymbol{\omega} d A\right)+\int_{D} \mathbf{l} \times \boldsymbol{\omega} d A \\
& \mathbf{A}=-b^{2}\left(\Omega A_{B}+\frac{1}{2} \int_{D} \boldsymbol{\omega} d A\right)-\frac{1}{2} \int_{D} l^{2} \boldsymbol{\omega} d A-\int_{B} l^{2} \Omega d A-\int_{D} \mathbf{l} \cdot \mathbf{b} \boldsymbol{\omega} d A .
\end{aligned}
$$

As in $3 \mathrm{D}$, the terms within parentheses can be combined into a well-defined integral $\int_{D \cup B} \omega d A$. This follows from Stokes theorem, $\int_{\tilde{D}} \boldsymbol{\omega} d A=-\oint_{\partial \tilde{D}} \mathbf{n}_{i} \times \mathbf{u} d s$, and invoking the no-slip boundary condition again which makes $\mathbf{n}_{i} \times \mathbf{u}$ continuous on the body-fluid boundary. Since $\int_{D \cup B} \omega d A$ $=-\oint_{S} \mathbf{n}_{i} \times \mathbf{u} d s$ it follows that the combined (fluid + solid) vorticity term is zero if the circulation at infinity is zero. Further, examining the limiting form of the second of the shear stress integrals (18) for the case $\int_{D \cup B} \omega d A \neq 0$, it is found that

$$
\begin{aligned}
\lim _{S \rightarrow \infty} \oint_{S}\left(\mathbf{x} \times(\nabla \mathbf{u}) \cdot \mathbf{n}_{i}\right) d s & =\lim _{S \rightarrow \infty} \oint_{S}\left(\mathbf{l} \times(\nabla \mathbf{u}) \cdot \mathbf{n}_{i}\right) d s, \\
& =-2 \int_{D \cup B} \boldsymbol{\omega} d A .
\end{aligned}
$$

The last line is obtained after noting that to leading order $\mathbf{u}$ is of the form $\mathbf{e}_{\theta} \int_{D \cup B} \omega d A /(2 \pi \hat{R})$ when $S$ is taken to be a circle of radius $\hat{R}$ centered at the body centroid and $\left(\mathbf{e}_{r}, \mathbf{e}_{\theta}\right)$ are unit vectors in a polar coordinate system.

To summarize, if the circulation at infinity is zero then

$$
\begin{aligned}
& \mathbf{L}=\int_{D} \mathbf{l} \times \boldsymbol{\omega} d A \\
& \mathbf{A}=-\frac{1}{2} \int_{D} l^{2} \boldsymbol{\omega} d A-\int_{B} l^{2} \Omega d A-\int_{D} \mathbf{l} \cdot \mathbf{b} \boldsymbol{\omega} d A
\end{aligned}
$$


are conserved by the dynamics. If not, then $\mathbf{L}$, as given by (28), is still conserved by the dynamics but $\mathbf{A}$, as given by (29) need not be.

Remark. It may be noticed that in these derivations the assumption of homogeneous density is made and this is because the density variations in the weakly compressible numerical scheme are negligible. In the simulations, density variations are typically bounded by $1 \%$.

Nevertheless if density variations in the fluid are significant then the invariant quantities have slightly different forms. For example, the linear impulse in 3D takes the form

$$
\mathbf{L}=\left(\varrho_{B}-\varrho_{0}\right) V_{B} \mathbf{U}+\frac{1}{2} \varrho_{0} \int_{D} \mathbf{l} \times \omega d V
$$

on the assumption that $\varrho \rightarrow$ constant $=\varrho_{0}$ as $S \rightarrow \infty$ at a sufficiently rapid rate.

\section{Cylinder translating with constant velocity: 2D and 3D}

The behavior of the far-field terms $\mathbf{F}_{S}$ and $\mathbf{M}_{S}$ is quite independent of whether the cylinder is in motion or at rest. It follows that in the case when the cylinder is moved with a constant translational speed $\mathbf{U}_{0}$ (possibly zero) the momentum relations turn out to be: (a) in $3 \mathrm{D}$,

$$
\begin{aligned}
\mathbf{F} & =\frac{d}{d t}\left(\frac{1}{2} \int_{D} \mathbf{1} \times \omega d V\right), \\
\mathbf{M} & =\frac{d}{d t}\left(-\frac{1}{2} \int_{D} l^{2} \omega d V-\int_{D} \mathbf{1} \cdot \mathbf{b} d V\right)
\end{aligned}
$$

(under Main assumption which implies $\int_{D} \omega d V=0$ ) and (b) in 2D,

$$
\begin{aligned}
\mathbf{F} & =\frac{d}{d t}\left(\int_{D} \mathbf{l} \times \omega d A+\mathbf{U}_{0} t \times \int_{D} \omega d A\right), \\
\mathbf{M} & =\frac{d}{d t}\left(-\frac{1}{2} \int_{D} l^{2} \omega d A-\left(2 v t+\mathbf{U}_{0}^{2} t^{2}\right) \times \int_{D} \omega d A-\int_{D} \mathbf{l} \cdot \mathbf{b} d A\right),
\end{aligned}
$$

where $\mathbf{F}$ and $\mathbf{M}$ are the external force and moment (about the center) required to translate the cylinder. In fact, the above two formulas continue to hold even if the translational velocity of the cylinder is not a constant-in this case the term $\mathbf{U}_{0} t$ is replaced by $\mathbf{b}(t)$. The force and moment expressions in this subsection agree with those obtained by $\mathrm{Wu},{ }^{50}$ for example, and even generalize them by accounting for possible non-zero values of $\int_{D} \omega d A$ in $2 \mathrm{D}$.

\section{RESULTS AND DISCUSSION}

We now turn to discuss the results for the interaction of a vortex pair with a freely moving neutrally buoyant circular cylinder. We will compare these findings with those results of the fixed circular cylinder case to highlight the differences caused by the free motion of the cylinder. Similar to earlier studies of Orlandi ${ }^{31}$ and Verzicco et al. ${ }^{48}$ investigating the interaction of a vortex pair with a fixed cylinder, we initialize the flow field with the vortex dipole discussed in Lamb ${ }^{23}$ and Batchelor, ${ }^{3}$ which has a vorticity distribution $\omega_{z}=k^{2} \psi$ within a circular recirculation zone with radius $a$, where $\psi$ denotes the streamfunction and $k$ is a parameter to be specified in the following. Homa et al. ${ }^{19}$ showed that this specification of the vorticity distribution corresponds well to that of a vortex pair generated experimentally from a planar nozzle. In an inviscid flow, this vortex pair translates at a velocity $u_{c}$. Outside of the recirculation zone, the flow field is assumed to be inviscid and described by the potential flow past a circular cylinder with radius $a$, which moves at the translation velocity of the vortex pair $u_{c}$ relative to the stationary frame of reference. The streamfunction possesses the form

$$
\begin{cases}\psi=C J_{1}(k r) \sin \theta-u_{c} r \sin \theta, & r \leq a, \\ \psi=-u_{c} \frac{a^{2}}{r} \sin \theta, & r>a,\end{cases}
$$




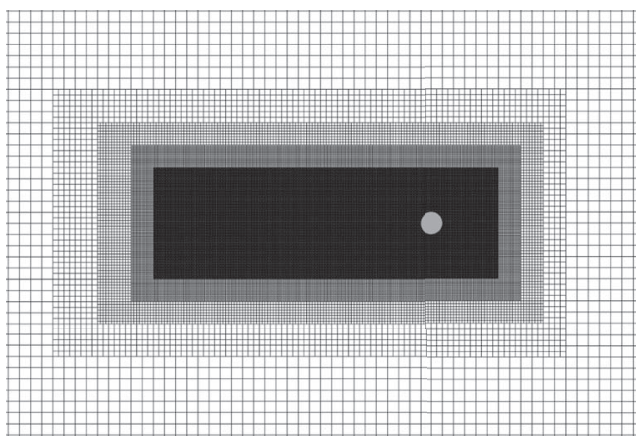

(a)

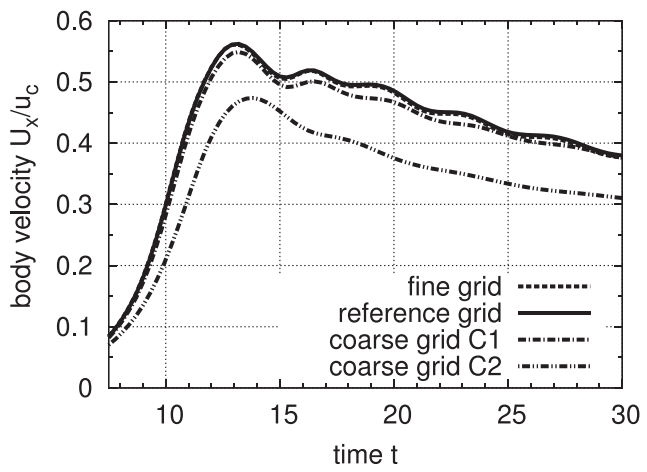

(b)

FIG. 4. Grid refinement study for the neutrally buoyant cylinder case: (a) part of the reference grid (not the entire far-field region is shown) and (b) normalized body velocity obtained for the neutrally buoyant cylinder case on the different grids. The fine-grid solution is nearly indistinguishable from the reference-grid solution.

where $J_{1}$ is the Bessel function of the first order and the $(r, \theta)$ coordinates are taken relative to the center of the vortex pair, which is initially positioned at $\left(x_{v}, y_{v}\right)=(-10 D, 0)$ where $D$ is the diameter of the cylinder. This position was determined based on preliminary numerical experiments to ensure that the vortex pair is initially undisturbed by the presence of the cylinder and vice versa. The parameter $k$ is chosen such that $J_{1}(k a)=0$. Using the first zero of $J_{1}$ gives $k=3.83 / a$. The vortex cores are located where $J_{1}$ possesses its first maximum, i.e., at $R=1.8412 / k$, where $R$ defines the radius of the vortex pair. The parameter $C$ is prescribed to match the inner and outer solutions at $r=a$.

The Reynolds number based on the translation velocity $u_{c}$ of the vortex pair and the cylinder diameter is $R e_{D}=500$ to match the simulation parameters used by Verzicco et al. ${ }^{48}$ The Mach number corresponding to $u_{c}$ is prescribed as $M=0.05$ to obtain results that are comparable to simulations of incompressible flows. As stated above, only small density variations of up to $1 \%$ were observed in the simulations. The cylinder is taken to be neutrally buoyant, i.e., $m_{\text {red }}=\pi / 4$. Initially, the circular cylinder is located far away from the vortex pair such that the flow field in the vicinity of the cylinder is nearly quiescent. At $t=0$, the center of the cylinder at diameter $D=2 R$ is positioned at $(X, Y)=(0,0)$. The computational grid covering the domain $\mathcal{D}:[-40 D, 24 D] \times[-32 D, 32 D]$ is locally refined such that the cylinder and the vortex pair are located in the finest mesh areas at all times and sponge layers are used at all far-field boundaries. To minimize the influence of boundary conditions the analysis of the flow fields using the expressions derived in Sec. IV is performed in a sub-domain $\widetilde{D}:[-20 D, 10 D] \times[-10 D, 10 D]$. Figure 4 (a) shows part of the computational grid used for the neutrally buoyant cylinder case simulations. The refinement patches are enlarged in the $y$ direction for the fixed cylinder case to capture the entire vortex motion on the finest grid patch. Figure 4(b) summarizes the results of a grid convergence study performed for the neutrally buoyant cylinder case. Clearly, the solutions on the reference grid, which is shown in Figure 4(a), are grid-converged such that this grid is used for all the simulations to be analyzed. On this grid, the mesh size varies from $\Delta x=0.5 D$ in the far-field to $\Delta x=0.03125 D$ in the fine mesh areas covering the vortex-pair-cylinder interaction.

The remaining of the section is organized as follows. In Sec. V A, the interaction of a vortex pair with a fixed cylinder is reviewed first. The observed vortex dynamics is next compared to the case in which the cylinder is neutrally buoyant and unrestrained. The forces on the fixed cylinder and the motion of the neutrally buoyant cylinder are related to the momentum expressions derived in Sec. IV. The fixed cylinder case and the neutrally buoyant cylinder case can be considered canonical problems of vortex-body interaction for the cases of a body maintaining its position in a vortical flow field and a body passively floating in the absence of external forces. In Sec. V B, a cylinder translating with constant velocity is considered, which is representative of constant-speed locomotion. The thrust required for this type of locomotion is again related to the conserved quantities derived in Sec. IV. 
TABLE I. Overview of the test cases.

\begin{tabular}{|c|c|c|}
\hline Case & Section & Remark \\
\hline Fixed cylinder & V A & $\begin{array}{l}\text { Uncoupled vortex-body interaction } \\
\text { body maintains its position }\end{array}$ \\
\hline Neutrally buoyant cylinder & V A & $\begin{array}{l}\text { Fully coupled vortex-body interaction } \\
\text { body floats passively in the fluid }\end{array}$ \\
\hline $\begin{array}{l}\text { Cylinder translating with } \\
\text { constant velocity }\end{array}$ & V B & $\begin{array}{l}\text { Uncoupled vortex-body interaction } \\
\text { body moves steadily }\end{array}$ \\
\hline $\begin{array}{c}\text { Neutrally buoyant cylinder } \\
\text { with constant thrust }\end{array}$ & V C & $\begin{array}{l}\text { Fully coupled vortex-body interaction } \\
\text { time-dependent motion of the self-propelling body }\end{array}$ \\
\hline Asymmetric fixed cylinder & V D & $\begin{array}{l}\text { Multi-dimensional uncoupled vortex-body interaction } \\
\text { body maintains its position }\end{array}$ \\
\hline $\begin{array}{l}\text { Asymmetric neutrally } \\
\text { buoyant cylinder }\end{array}$ & V D & $\begin{array}{l}\text { Multi-dimensional coupled vortex-body interaction } \\
\text { body floats passively in the fluid }\end{array}$ \\
\hline
\end{tabular}

Section V C presents the case of locomotion with constant thrust, while the body is subjected to the fluid forces. Finally, the theoretical expressions are verified for a fully multi-dimensional case in Sec. V D, where simulation results of an asymmetric interaction of a vortex pair with a circular cylinder are discussed. An overview of the test cases is provided in Table I.

\section{A. The fixed cylinder case and the neutrally buoyant cylinder case}

\section{Description of the flow field}

As a baseline case, we resimulated the interaction of a vortex with a fixed cylinder. ${ }^{16}$ Note that the current numerical method as well as the initial set-up of the problem are slightly different from the method used in Hartmann et al. ${ }^{16}$ We briefly summarize the main features of the standard cylinder flow field to more evidently discuss the differences to the moving and asymmetric flow problems. The vorticity field is illustrated at two instants in Figure 5. As the vortex pair approaches the cylinder, strong secondary vorticity of opposite sign is observed in the boundary layers on the windward side of the cylinder surface, Figure 5(a). At $t=12$ shown in Figure 5(b), the boundary layers are separated and the secondary vorticity rolls up into vortices on either side of the cylinder which pair with vortices

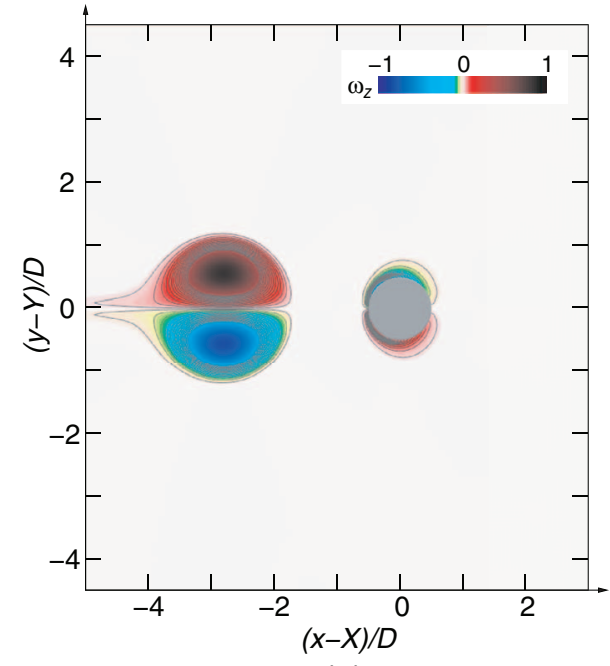

(a)

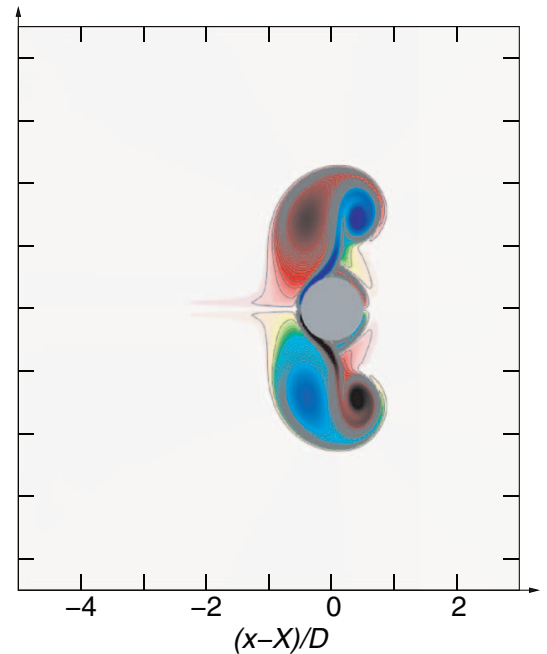

(b)

FIG. 5. Interaction of a vortex dipole with a fixed circular cylinder at $R e_{D}=500$. Thirty vorticity contours in the range $-0.2 \leq \omega_{z} \leq 0.2$ are plotted at (a) $t=8$ and (b) $t=12$. 


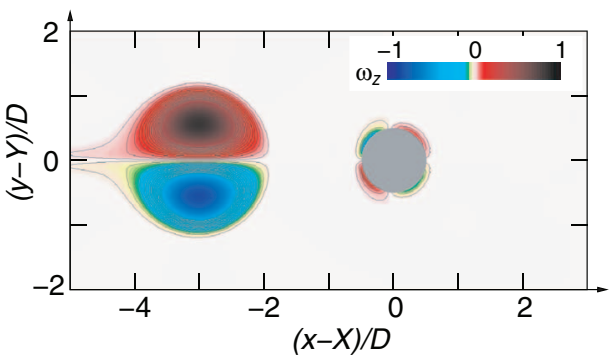

(a)

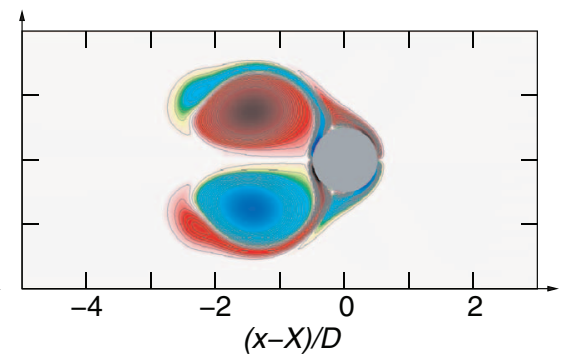

(b)

FIG. 6. Interaction of a vortex dipole with a neutrally buoyant circular cylinder at $R e_{D}=500$. Thirty vorticity contours in the range $-0.2 \leq \omega_{z} \leq 0.2$ are plotted in a moving frame of reference at (a) $t=8$ and (b) $t=14$.

originating from the disintegrated primary vortex pair to form two opposite-signed vortex pairs. As the flow develops, the newly formed vortex pairs travel upstream in a semi-circular motion.

It is interesting to note that the interaction of a vortex pair with a fixed cylinder can also be considered a model for the interaction of the wake vortices initially attached to a suddenly decelerated cylinder with the body itself. Indeed, the present results show a striking similarity to the experimentally and numerically obtained flow fields reported by Sheard et al. ${ }^{44}$ As the cylinder is decelerated the symmetric wake vortices attached to it are pushed around the cylinder generating secondary vorticity at its surface. For sufficiently developed wake vortices and at a comparably large Reynolds number-in the study of Sheard et al. ${ }^{44}$ the Reynolds number is defined based on the cylinder diameter and its velocity before deceleration - as that used in the present study the wake vortices and the secondary vorticity form two vortex pairs that travel backwards on a semi-circular path much like it is observed for the interaction of a vortex pair with a fixed cylinder in Figure 5.

In the case where the cylinder is free to move in response to the fluid force, the vortex dynamics is quite different as Figure 6 illustrates. As the vortex pair approaches the cylinder, a smaller amount of secondary vorticity is created in the boundary layer on the windward side-we will assume "windward" and "leeward" to still refer to the same sides as in the fixed cylinder case-of the cylinder surface than in the fixed cylinder case. However, at the same time secondary vorticity of the same sign is seen in the boundary layers on the leeward side which are formed due to the motion of the cylinder from left to right. This becomes evident by comparing frame (a) in Figures 5 and 6 . The approaching vortex pair accelerates the cylinder as shown in Figure 7, which illustrates among other

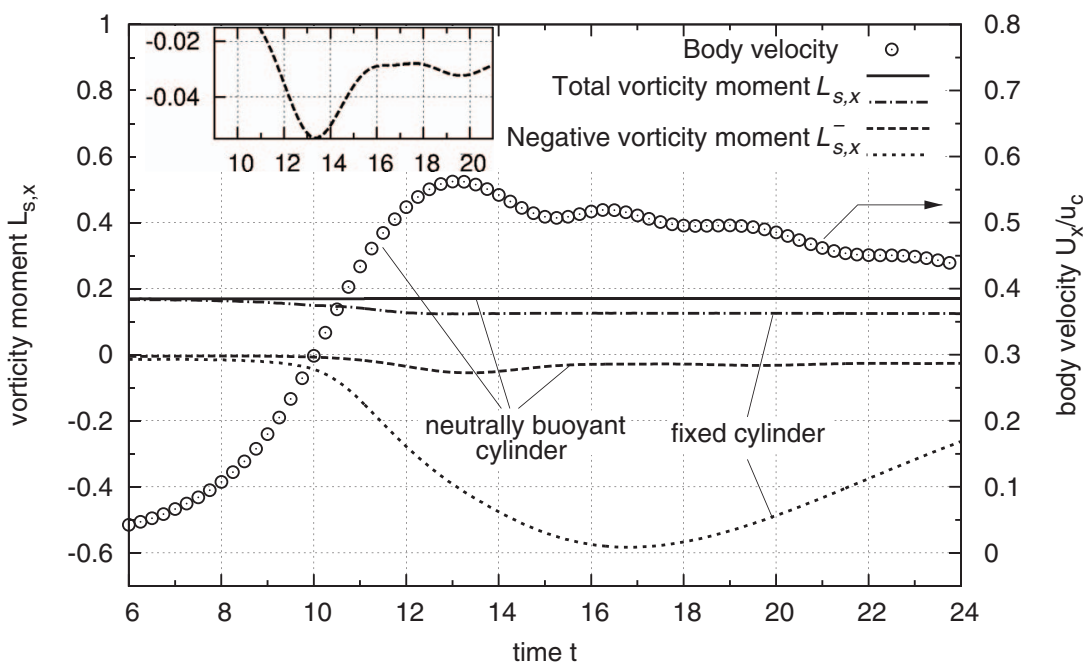

FIG. 7. Interaction of a vortex dipole with a circular cylinder at $R e_{D}=500$; comparison of the vorticity moment vs time for the cases of a fixed and a neutrally buoyant cylinder. The inset shows a zoom of the negative vorticity moment for the case of the neutrally buoyant cylinder. 


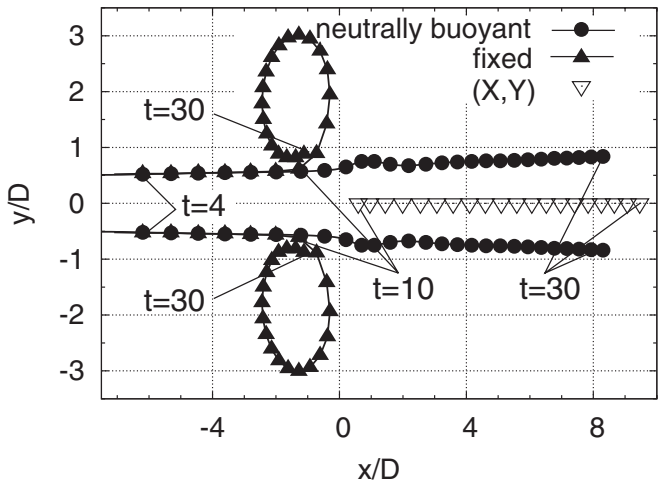

(a)

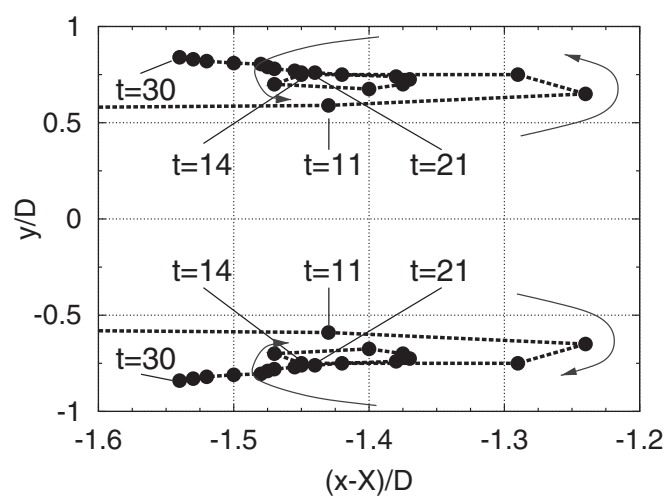

(b)

FIG. 8. Path lines of the vortex cores for the interaction of a vortex dipole with a neutrally buoyant circular cylinder at $R e_{D}$ $=500$ : (a) absolute location for the neutrally buoyant and the fixed cylinder cases and location of the cylinder center of mass $(X, Y)$ and (b) location relative to the position $(X, Y)$ of the neutrally buoyant cylinder center. Solutions are plotted for each non-dimensional time unit.

results the cylinder velocity over time. Figure 6(b) shows that the patches of secondary vorticity from the separating boundary layers are wrapped around the primary vortex pair and do not form a coherent vortex which pairs with the primary vortex as in the fixed cylinder case. Moreover, the primary vortex seems to maintain a fairly constant position with respect to the moving cylinder. For a more detailed discussion of the flow field, we refer the interested reader to Hartmann et al. ${ }^{16}$ The major difference in secondary vortex formation and subsequent pairing is quite likely due to the stream, from right to left, relative to the moving cylinder, caused by the (unsteady) motion of the cylinder. It will be shown in Sec. V B that superimposing a steady stream from right to left, which is equivalent to a steady cylinder motion from left to right, can also alter the vortical events in the fixed cylinder case.

To further elucidate the differences in the vortex dynamics in both cases, the path lines of the cores of the primary vortex pair are plotted in Figure 8. First, Figure 8(a) shows the position of the primary vortex cores for both the neutrally buoyant cylinder and the fixed cylinder and the location of the neutrally buoyant cylinder center of mass. The time interval between each data point is one time unit. In the neutrally buoyant cylinder case the translation velocity of the vortex cores significantly decreases at $t=12$, which is evident from the narrower spacing between the data points in Figure 8(a). At this time level, the cylinder has almost reached its maximum velocity as shown in Figure 7. At later times, Figure 8(a) suggests that the vortex cores and the cylinder center of mass maintain a fairly constant distance in the streamwise $x$ direction. This is more clearly shown in Figure 8(b), which plots the position of the primary vortex pair relative to the cylinder center of mass in the neutrally buoyant cylinder case. Between time levels $t=11$ and $t=30$ the vortex cores are located at a distance of $1.2 D-1.6 D$ to the cylinder center on its windward side. While the vortex cores and the cylinder move at a similar velocity, the vortex cores slowly move apart from each other as shown in Figure 8(a). The significant differences in the vortex dynamics upon interaction with the cylinder in the fixed and neutrally buoyant cylinder cases, which became apparent in the discussion above (compare, e.g., Figure 6(b) for the neutrally buoyant cylinder case with Figure 5(b) for the fixed cylinder case) are also corroborated in Figure 8(a), which clearly shows the semi-circular path and bounce-back of the primary vortex pair in the fixed cylinder case in contrast to the predominantly translational motion in the neutrally buoyant cylinder case.

Figure 8(b) clarifies that even though the primary vortex pair remains attached to the moving cylinder, it performs a small-scale circular motion between time levels $t=14$ and $t=21$, which resembles the much larger semi-circular path of the primary vortex pair in the fixed cylinder case. Along with slow transverse motion of the vortex cores observed in Figure 8(a) the cores also slowly move away from the cylinder. This, however, can be attributed to viscous diffusion resulting in decreasing vortex strength and an increasing vortex size. 


\section{Correlation of vortex dynamics and cylinder behavior}

The motion of the cylinder is characterized in Figure 7, where among other quantities the streamwise velocity $U_{x}$ of the cylinder normalized by the translational velocity $u_{c}$ of the vortex pair is plotted vs time. Initially, the cylinder monotonically accelerates until the vortex pair reaches the cylinder at $t=13$, i.e., shortly before the time level shown in Figure 6(b). After reaching its peak value, the cylinder velocity slowly decreases. However, this decrease is not monotonic and the velocity plot exhibits several humps, indicating the unsteady interaction between the vorticity field and the movement of the cylinder.

Likewise, examining the total vorticity moment in a spatially fixed frame with origin at $(x / D$, $y / D)=(0,0)$,

$$
\mathbf{L}_{s}:=\int_{\mathcal{D}} \mathbf{x} \times \omega d A,
$$

where $\mathbf{x}$ denotes the position vector in this frame, shows that it is conserved in the numerical simulation. The actual maximum variation of $L_{s, x}$ in the numerical simulation is $1 \%$. This slight variation is due to the initial transient in the numerical solution and the finiteness of the computational domain. After time level $t=8$ when the strong interaction between the vortex pair and the neutrally buoyant cylinder begins, the maximum relative variation of $L_{s, x}$ is $7 \times 10^{-4}$. The conservation of the total vorticity moment is consistent with the fact that for this symmetric vorticity distribution the uni-directional vector $\mathbf{L}_{s}=\mathbf{L}+\mathbf{b} \times \int_{\mathcal{D}} \omega d A=\mathbf{L}$ is invariant as discussed by the analysis of Sec. IV. For the fixed cylinder case, $\mathbf{L}_{s}$ shows a significant decrease between times $t=8$ and $t=12$. In view of Eq. (33) (with $\int_{D} \omega d A=0$ ), this can be explained by the force exerted on the fluid by the cylinder to maintain its position. This external force acts in the $-x$ direction and hence, results in a decrease of the total vorticity moment. Indeed, Figure 9 shows that the external force coefficient $C_{x, e x t}=-C_{x}$, i.e., the non-dimensional negative drag force appearing in Eq. (7), correlates nearly perfectly with the non-dimensional time derivative of $L_{s, x}$,

$$
\frac{d L_{s, x}}{d t}=\frac{1}{\varrho u_{\infty}^{2} D} \frac{d L_{s, x}^{*}}{d t^{*}},
$$

where the asterisk identifies dimensional quantities. This correlation

$$
2 \frac{d L_{s, x}}{d t}=C_{x, e x t}
$$

is a key result of the present study. It shows that external forces on a body, i.e., the force exerted on the fluid by the body such as the thrust of a self-propelling body, can be obtained from a measurement of the velocity and vorticity fields. In this context, the fixed cylinder case can be considered a model for a self-propelling body maintaining its position. As for the example of a swimming fish, the resulting

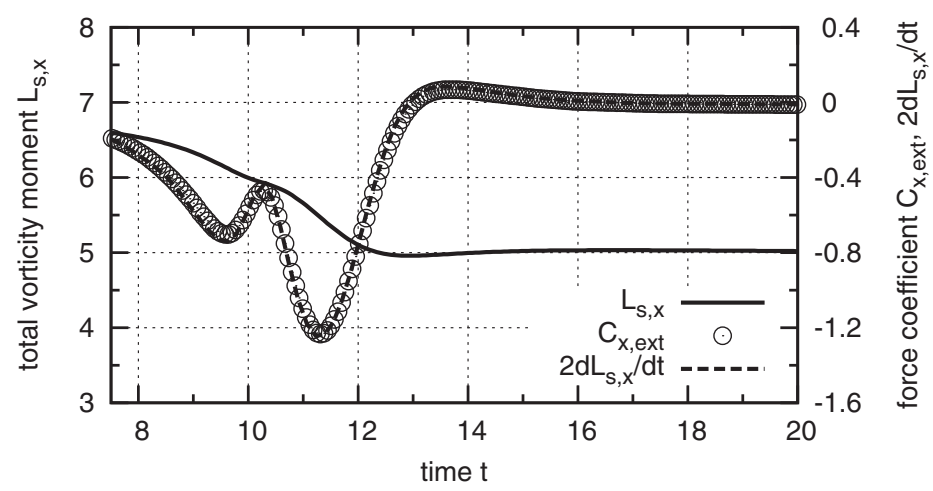

FIG. 9. Interaction of a vortex dipole with a fixed cylinder. Correlation of the external force coefficient $C_{x, e x t}$ and the time variation $d L_{s, x} / d t$ of the total vorticity moment. 


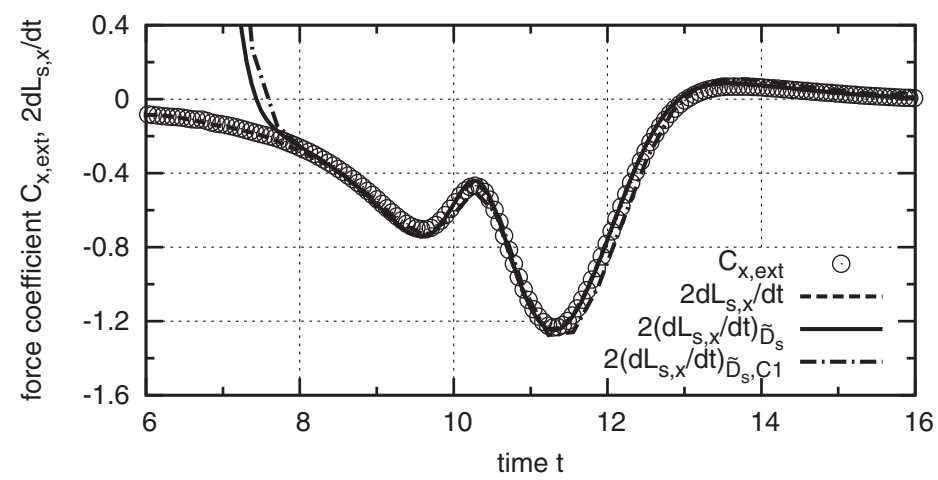

FIG. 10. Interaction of a vortex dipole with a fixed cylinder. Correlation of the external force coefficient $C_{x, e x t}$ and the time variation $d L_{s, x} / d t$ of the total vorticity moment for different measurement domains and grid resolutions.

force, i.e., the thrust generated by the fish minus the fluid drag, is zero and can be easily deduced from experimental data of the body motion. The correlation given by Eq. (36) allows to compute the external force exerted on the fluid by the fish, i.e., the thrust, and hence also the drag force given the easily obtainable resulting force. These quantities are otherwise difficult to measure experimentally. In this regard, the present result can be understood to represent a bounded and discrete data set of the flow velocity field, which could be obtained in an experiment by, e.g., particle-image velocimetry measurements. Such correlations in experiments have indeed been attempted previously. 2,25,30

Note that the equivalence of negative external force and drag force does not hold for the neutrally buoyant cylinder. The neutrally buoyant cylinder is exposed to non-zero drag which causes its acceleration but the external force is zero at all times. Hence, it should be emphasized that in general the correlation shown in Figure 9 refers to the external force applied to the body and not to the fluid force.

In terms of using the correlation given by Eq. (36) in an experiment it is crucial to know the required size of the measurement volume and the resolution at which the velocity field must be recorded. In other words, one may ask the question: How sensitive is the correlation shown in Figure 9 with respect to a decrease of the measurement domain and a decrease of the grid resolution? A coarser grid also introduces larger discretization errors into the numerical solution, which corresponds to a reduced accuracy. In terms of an experiment, this is the analogue of increased measurement errors. Figure 10 compares the results plotted in Figure 9 with data measured in a smaller rectangular domain $\widetilde{D}_{s}:[-4.375 D, 1.875 D] \times[-4.6875 D, 4.6875 D]$ around the cylinder on the reference grid and on the coarse grid $C 1$. The size of $\widetilde{D}_{s}$ is chosen such that $200 \times 300$ velocity vectors can be recorded at the finest resolution on the reference grid. On the coarse grid $\mathrm{C} 1$, this corresponds to 100 $\times 150$ velocity vectors. The results shown in Figure 10 evidence that the coarse-grid resolution and the small measurement domain $\widetilde{D}_{s}$ are sufficient to accurately determine the forces on the cylinder from the velocity and vorticity fields. Considering a prospective experimental investigation this 100 $\times 150$ velocity field can be achieved using high-speed particle-image velocimetry (PIV) and, e.g., a Photron Fastcam SA- 2 camera at a resolution of $1472 \times 2048$ pixels. To ensure the necessary number of velocity vectors within the measurement area $6.25 D \times 9.375 D$ the size of the interrogation spots is approximately $24 \times 24$ pixels with an overlap factor of $50 \%$. At this resolution, the camera provides a recording rate of $1.5 \mathrm{kHz}$ leading to an adequate sampling rate for the PIV images of $750 \mathrm{~Hz}$. That is using standard equipment it is possible to capture the spatial and temporal variation of the flow field at a resolution that is sufficient to apply Eq. (36). Note that this statement holds only for the time frame in which all significant vortical structures are contained in the measurement or computational volume, and that this requirement applies to the general, asymmetric case. It goes without saying that in a symmetric two-dimensional case it is sufficient for the domain to capture one half of the flow field. As Figure 5(a) shows, the primary vortices have just entered the small measurement domain $\widetilde{D}_{s}$ at time level $t=8$, which explains why the curves in Figure 10 representing the data recorded in the domain $\widetilde{D}_{s}$ significantly deviate from those based on data from $\widetilde{D}$ in the time frame between 
$t=6$ and $t=8$. Note that the accuracy requirements to use Eq. (36) are very specific to the experimental and numerical methodology and before a general statement can be made further investigations are to be performed.

It is also interesting to note the behavior of the negative vorticity moment in the spatially fixed frame,

$$
\begin{aligned}
L_{s, x}^{-} & :=\int_{\mathcal{D}^{-}}(\mathbf{x} \times \boldsymbol{\omega}) \cdot \mathbf{i} d A, \\
& =\int_{\mathcal{D}^{-}} y \omega_{z} d A .
\end{aligned}
$$

In Eq. (37), $\mathcal{D}^{-}$represents those cells of $\mathcal{D}$ in which $(\mathbf{x} \times \boldsymbol{\omega}) \cdot \mathbf{i}<0$, where $\mathbf{i}$ is the unit vector in the $x / D$-direction. It follows that $\mathcal{D}^{-}$is the domain of the negative vorticity in the upper half-plane and the positive vorticity in the lower half-plane. Similarly, a positive vorticity moment, $L_{s, x}^{+}$, can be defined. Since $\mathcal{D}=\mathcal{D}^{+} \cup \mathcal{D}^{-}$, it follows that in the general three-dimensional case

$$
\mathbf{L}_{s}=\mathbf{L}_{s}^{+}+\mathbf{L}_{s}^{-}=\text {invariant. }
$$

The total and negative vorticity moments are plotted for the fixed cylinder case and the neutrally buoyant cylinder case in Figure 7. Clearly, in the fixed cylinder case the larger $+y /-y$ excursions of the secondary (negative/positive) vortices compared to the neutrally buoyant cylinder case, which can be observed by comparing Figures 5 and 6 , result in lower values of $L_{s, x}^{-}$.

Figure 11 shows a comparison of the total vorticity moment $(\mathbf{x} \times \boldsymbol{\omega})_{x}$ for the fixed cylinder case and the neutrally buoyant cylinder case along with the velocity vectors. The secondary shear layers generated in the fixed cylinder case make a negative contribution to $L_{s, x}$ and clearly are significantly stronger than those appearing in the neutrally buoyant cylinder case. As Figure 7 clearly shows, this results in a decreasing $L_{s, x}^{-}$, while $L_{s, x}^{+}$does not increase in the same way. In the neutrally buoyant cylinder case, the decrease in $L_{s, x}^{-}$should be compensated by an increase in $L_{s, x}^{+}$according to the invariance of $L_{s, x}$ stated in Eq. (39). This invariance is indeed observed in the numerical simulation of the neutrally buoyant cylinder case as shown in Figure 7. Moreover, the acceleration of the circular cylinder seems connected to the behavior of the negative vorticity moment. The initially monotonic increase of the cylinder velocity up to time level $t \approx 13$ goes along with a monotonic decrease of the negative vorticity moment. The same correspondence is observed in the period $t \approx 13-16$, in which the cylinder velocity decreases and the negative vorticity moment increases.

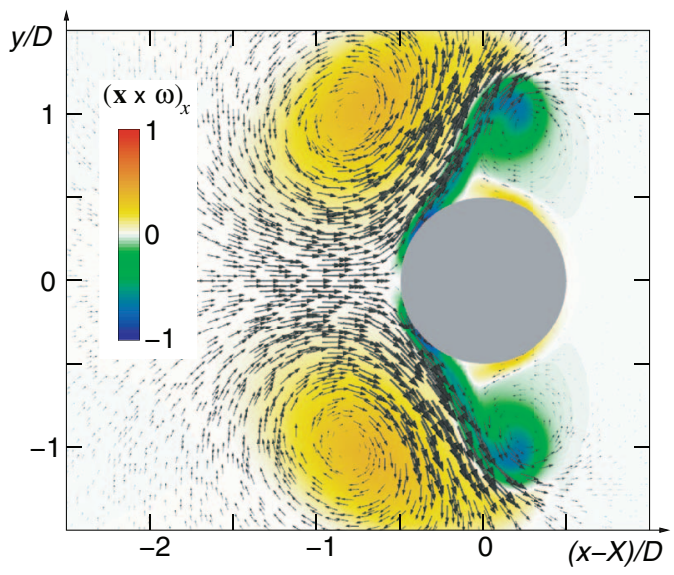

(a)

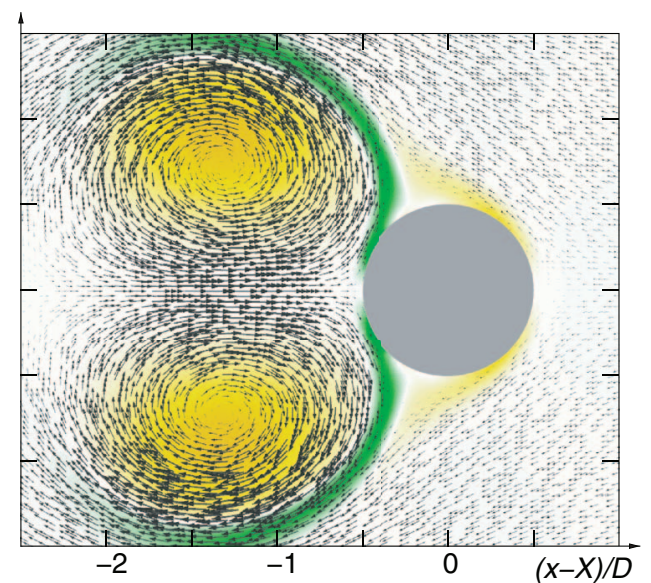

(b)

FIG. 11. Vorticity moment $(\mathbf{x} \times \omega)_{x}$ and scaled velocity vectors for instantaneous solutions of the flow at $\operatorname{Re}_{D}=500$ : (a) fixed cylinder at time level $t=11$ and (b) neutrally buoyant cylinder at time level 13, where the cylinder nearly reaches its maximum velocity, see Figure 7(c). 


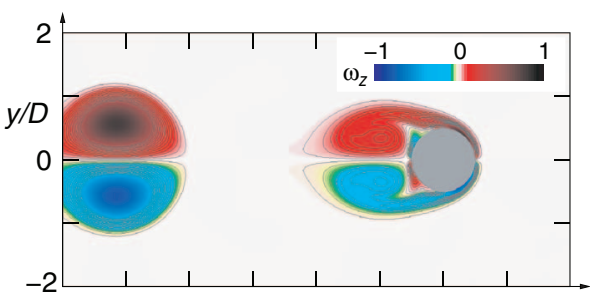

(a)

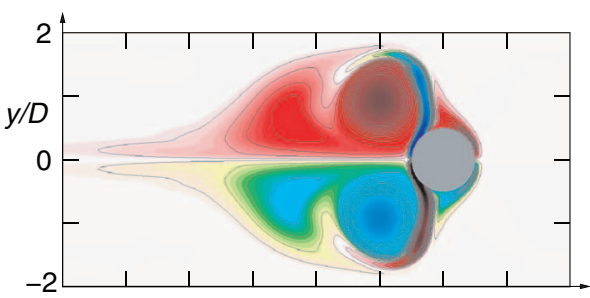

(c)

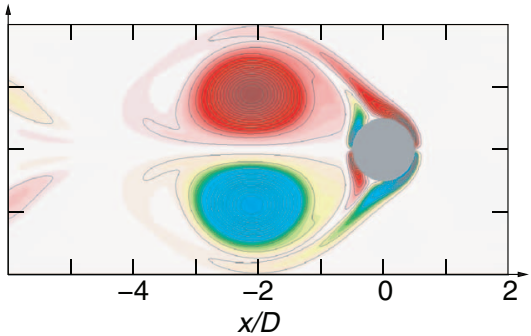

(e)

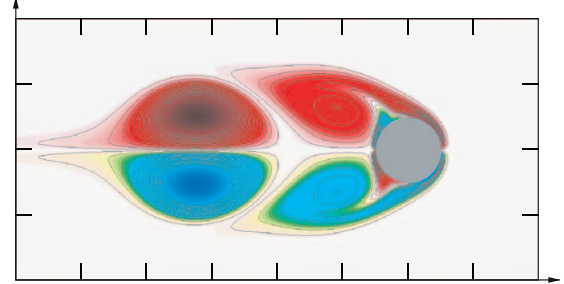

(b)

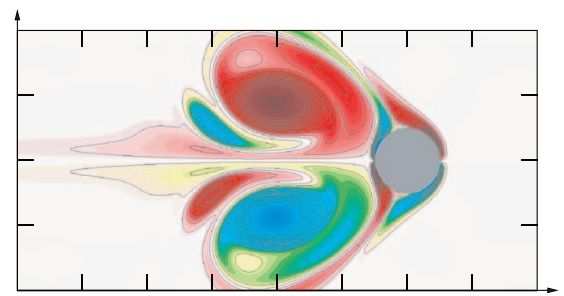

(d)

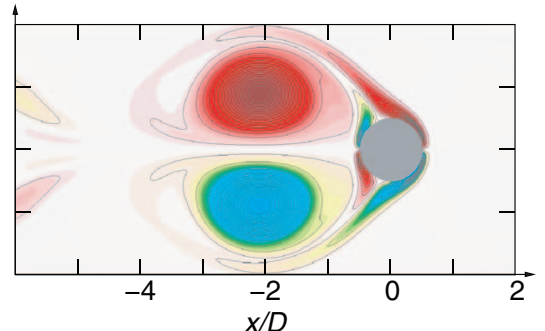

(f)

FIG. 12. Interaction of a vortex dipole with a circular cylinder translating at the constant speed $U_{x}=0.4 u_{c}$. Thirty vorticity contours in the range $-0.5 \leq \omega_{z} \leq 0.5$ are plotted in a moving frame of reference at (a) $t=10$, (b) $t=15$, (c) $t=20$, (d) $t=25$, (e) $t=30$, and (f) $t=35$.

\section{B. The cylinder translating with constant velocity case}

To understand the mechanism which causes the significant difference in the vortex dynamics between the fixed cylinder and the neutrally buoyant cylinder we next consider the interaction of a vortex pair with a circular cylinder translating with constant velocity. The cylinder moves at the constant speed $U_{x}=0.4 u_{c}$ in the $x$ direction, i.e., in the same direction as the vortex pair moves, but at a smaller velocity to allow an interaction between the vortex pair and the cylinder. Furthermore, as a comparison with Figure 7 shows, the cylinder velocity is smaller than that of the neutrally buoyant cylinder after time level $t=11$, i.e., shortly after the vortex pair has reached the cylinder. What distinguishes this test case from the fixed cylinder is (1) a smaller relative approach velocity of the vortex pair towards the cylinder and (2) a recirculation bubble on the windward side-we will assume "windward" and "leeward" to still refer to the same sides as in the fixed cylinder case—of the cylinder due to its translatory motion, which forms before the vortex pair interacts with the cylinder.

Figure 12 illustrates the evolution of the vorticity field over time. At time $t=10$ in Figure 12(a) a recirculation bubble which has formed on the windward side of the cylinder can be observed. As the vortex pair approaches the cylinder, the counterrotating wake vortices of the cylinder are separated and move away from each other in the cross-stream direction while they are still connected to the separating boundary layers of the cylinder as Figure 12(b) shows for time level $t=15$. Next, the wake vortices start to orbit around the same-signed primary vortex pair, which almost touches the cylinder and causes the generation of secondary vorticity on the windward side of the cylinder. Simultaneously, the wake vortices are disconnected from the detaching cylinder boundary layers and separated by the emerging layers of secondary vorticity. This three-layer structure of 


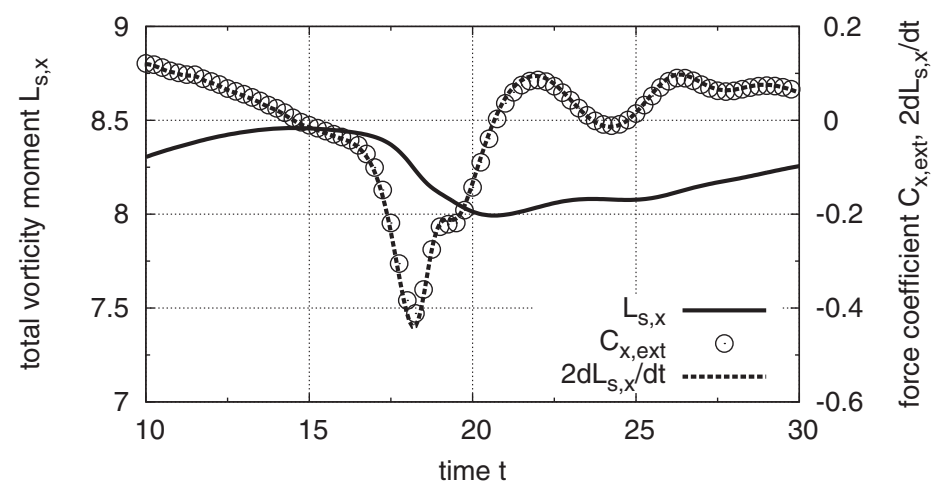

FIG. 13. Interaction of a vortex dipole with a circular cylinder which is moving at the constant speed $U_{x}=0.4 u_{c}$. Correlation of the external force coefficient $C_{x, \text { ext }}$ and the time variation $d L_{s, x} / d t$ of the total vorticity moment.

primary vortices, layers of secondary vorticity, and the separating boundary layers resembles the configuration which forms in the neutrally buoyant cylinder case. ${ }^{16}$ Between time levels $t=20$ and $t=25$ shown in Figures 12(c) and 12(d), respectively, the wake vortices appear to be sucked in by the primary vortex pair and finally coalesce with the same-signed primary vortices. The ensuing vortex dynamics is quite similar to that observed in the neutrally buoyant cylinder case. ${ }^{16} \mathrm{~A}$ layer of secondary vorticity is ejected from the cylinder surface and wraps around the primary vortices until parts of it form a secondary vortex pair as shown in Figure 12(d), which subsequently travels away from the cylinder. The ejection of secondary vorticity causes the primary vortices to rotate about their axes as a comparison of Figures 12(c)-12(e) suggests. After that, the vortex-pair-cylinder configuration appears to translate in a stable fashion with the vortex pair attached to the cylinder, Figures 12(e) and 12(f), which again resembles the neutrally buoyant cylinder case.

The results show that the characteristic pairing of primary and secondary vortices and the bounce-back of the primary vortex pair observed in the fixed cylinder case can be effectively suppressed by a simple imposed translatory motion of the cylinder. The resulting vortex dynamics resembles that observed in the neutrally buoyant cylinder case.

To relate these results to the theoretical expression for the body force required for uniform translation, the quantity $L_{s, x}$, its time derivative, and the coefficient of the external force $C_{x, e x t}$ applied to the circular cylinder are plotted in Figure 13. As for the fixed cylinder, the external force coefficient and the time derivative of $L_{s, x}$ correlate excellently in the numerical simulations as predicted by Eq. (33). Hence, the required thrust for constant-speed locomotion can be deduced from the vorticity field as given by the right-hand side of Eq. (33). Like in the fixed cylinder case, the external force is equivalent to the negative drag on the cylinder when it is moved at a constant speed. Note that in the present symmetric configuration the second term in parentheses in Eq. (33) drops out such that Eq. (33) reduces to

$$
\mathbf{F}=\frac{d}{d t} \int_{D} \mathbf{1} \times \omega d A
$$

It is interesting to compare Figures 13 and 9 to gain further insight into the differences between the various test cases. This shows the time variation of $L_{s, x}$ to be significantly smaller in the present case than in the fixed cylinder case, i.e., it is closer to the constant value in the neutrally buoyant cylinder case. As shown in Figure 9, $L_{s, x}$ attains a nearly constant value and correspondingly the drag coefficient is almost zero in the fixed cylinder case after time level $t=14$. This is the time at which the newly formed vortex pairs start to move away from the cylinder on a semi-circular path, Figure 5, and hence, the cylinder is not exposed to a significant streamwise flow any longer resulting in a near-zero drag. The smaller peak values of the fluid force on the cylinder translating with constant velocity than on the fixed cylinder explain the smaller amount of secondary vorticity generated on the windward side of the cylinder. This in turn results in the suppression of the vortex re-pairing and primary vortex bounce-back observed in Figure 12. 


\section{The neutrally buoyant cylinder with constant thrust case}

Next, the neutrally buoyant cylinder responds to the fluid forces, but a constant external force is added, such that elements of the fixed cylinder and the neutrally buoyant cylinder are combined. A constant non-dimensional thrust $F_{x}=-0.0025 \pi D^{2}$ is applied to the cylinder accelerating it in the $-x$ direction towards the approaching primary vortex pair. This case can be considered a model problem for constant-thrust locomotion. The computational set-up is similar to that for the neutrally buoyant cylinder case. The grid is extended in the $x$ direction to capture all the relevant vortex dynamics on the finest mesh.

Figure 14 shows the evolution of the vorticity field. At time $t=3$ shown in Figure 14(a) the cylinder has almost reached the vortex pair. A symmetric recirculation bubble, the vortices of which possess the opposite sign to the primary vortices, has formed in the cylinder wake, i.e., on the leeward side of the cylinder. This bubble interacts with the primary vortex pair as it passes the cylinder, Figure 14(b). In passing the cylinder the primary vortices are separated and entrain the secondary vortices forming the recirculation bubble away from the cylinder. This can be observed at time level $t=5$ illustrated in Figure 14(c). Subsequently, the primary vortices make the secondary vortices move, which have the opposite orientation. At time $t=6$ shown in Figure 14(d) parts of the secondary vorticity form a secondary vortex pair which travels back towards the cylinder due to its opposite orientation, while the other part of the secondary vorticity begins to roll around the primary vortices, which start a rotation about their axes. The secondary vortex pair is embraced again by the separating shear layers at time $t=7$ shown in Figure 14(e), while the rotating primary vortices move away from the cylinder dragging along a tail of secondary vorticity.

Unlike in the previously discussed fixed cylinder case and the case of the cylinder translating with constant velocity the external force does not exactly balance the drag at all times resulting in an unsteady motion of the cylinder. Due to the constant external force applied to the cylinder and the symmetry of the problem $L_{s, x}$ is expected to have a constant negative slope following Eqs. (33) and (40). Indeed, this is observed in the numerical simulations as Figure 15 clearly shows. Furthermore, secondary vorticity is generated in the wake of the moving cylinder as the plot for $L_{s, x}^{-}$shows. The behavior of the cylinder can again be related to the secondary vorticity as in the neutrally buoyant cylinder case. After an initial strong acceleration in the $-x$ direction until $t=1$, Figure 15 shows the cylinder velocity to only slightly decrease until $t=3$ shortly before the cylinder reaches the vortex pair, Figure 14(a). Between time levels $t=3$ and $t=4$ the cylinder slows down significantly, which goes along with a sharp increase of secondary vorticity, i.e., a drop of $L_{s, x}^{-}$, Figure 14(b). As soon as the vortex pair passes the cylinder centerline the cylinder accelerates again in the $-x$ direction and the $L_{s, x}^{-}$attains a nearly constant value. This supports what was observed in Figure 7 for the neutrally buoyant cylinder case.

\section{The asymmetric fixed and neutrally buoyant cylinder case}

Finally, we consider an asymmetric vortex-pair-cylinder interaction to show numerically the conservation of $\mathbf{A}$ as formulated in Eq. (31). In this test case the vortex pair is initially slightly offset in the vertical direction such that its center is located at $\left(x_{v}, y_{v}\right)=(-10 D, 0.1 D)$. The evolution of the vorticity field is illustrated in Figure 16. Figure 16(a) shows the field at $t=10$ shortly before the vortex pair reaches the circular cylinder. The asymmetric distribution of secondary vorticity on the windward and leeward side of the cylinder results from the asymmetrically positioned vortex pair and from the motion of the cylinder in the $+x$ direction and the $-y$ direction. The latter was not observed in the symmetric test cases and is shown by the distribution of the velocity component $U_{y} / u_{c}$ in Figure 17(a). During the interaction the circular cylinder is further accelerated in the $+x$ and $-y$ direction and the secondary vorticity generated on the windward side of the cylinder-we will assume "windward" and "leeward" to still refer to the same sides as in the fixed cylinder case—starts to wrap around the primary vortex pair as shown in Figure 16(b). At roughly $t=13$ the cylinder reaches its maximum streamwise velocity, Figure 17(a), after which it sharply drops. At $t=14$ shown in Figure 16(c), the positive secondary vorticity has moved around the negative primary vortex and has formed an isolated patch of vorticity disconnected from the cylinder. In contrast, the negative 
(a)

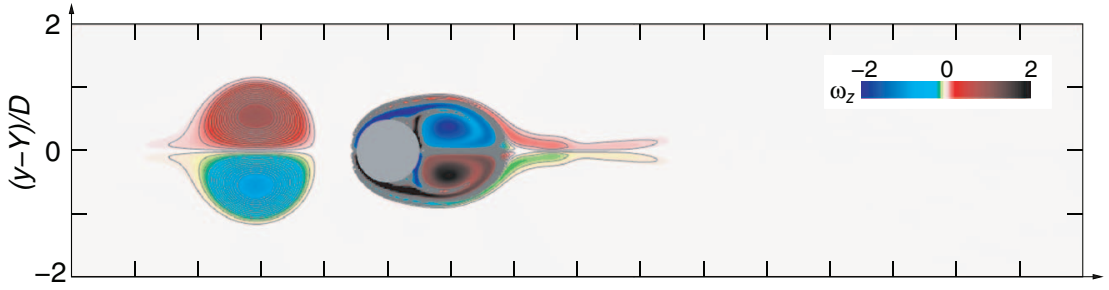

(b)

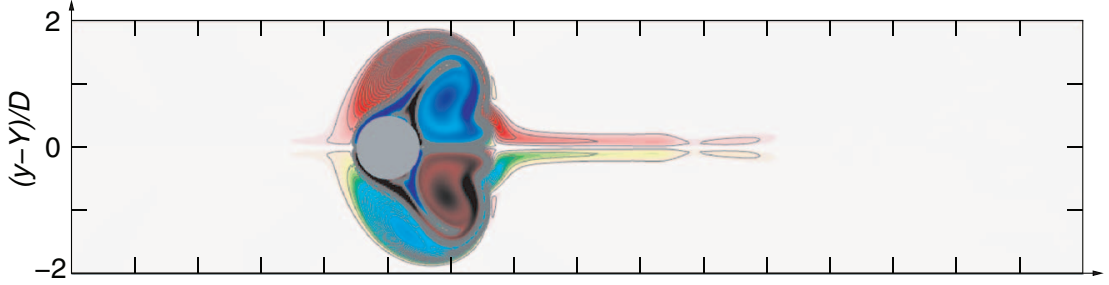

(c)

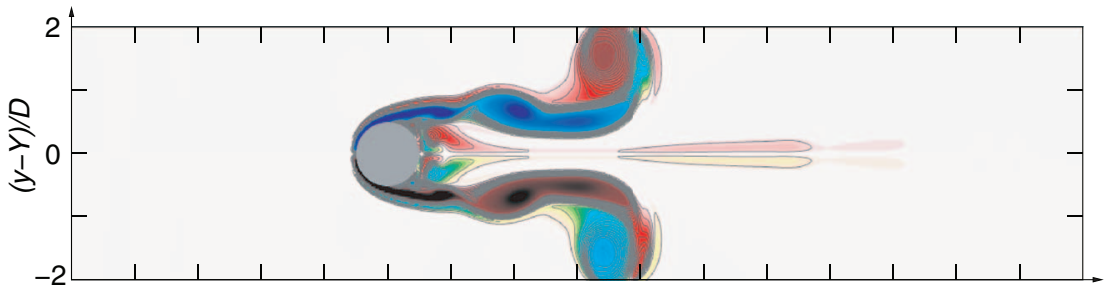

(d)

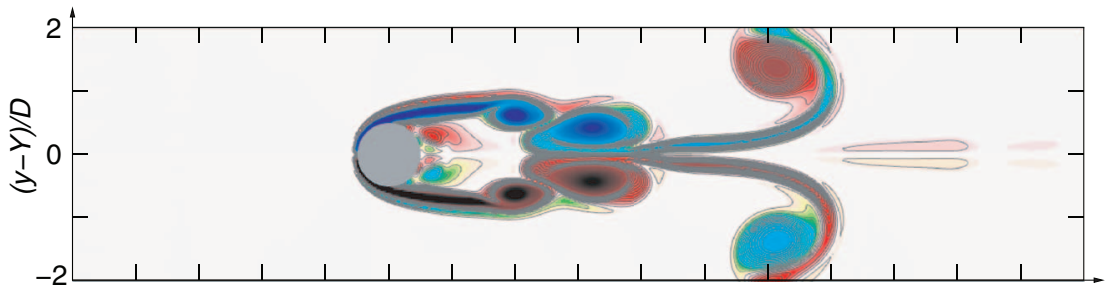

(e)

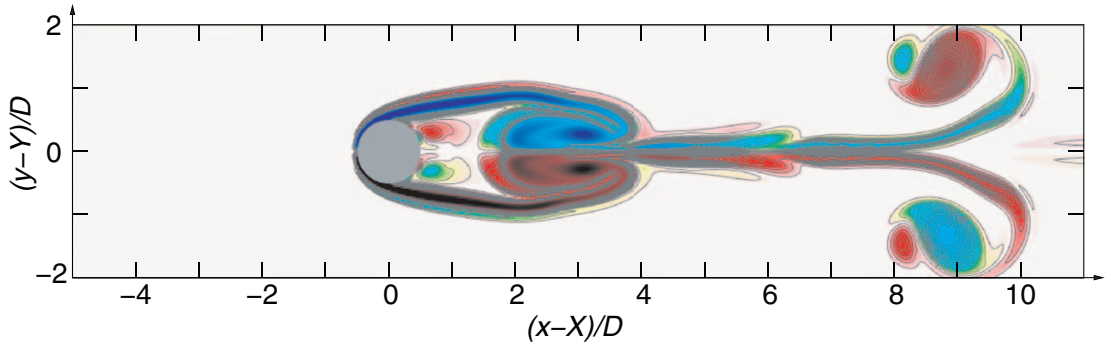

FIG. 14. Interaction of a vortex dipole with a neutrally buoyant circular cylinder subject to a constant thrust at $\operatorname{Re}_{D}=500$. A constant non-dimensional force $F_{x}=-0.0025 \pi D^{2}$ is imposed on the cylinder. Thirty vorticity contours in the range $-0.4 \leq \omega_{z} \leq 0.4$ are plotted at (a) $t=3$, (b) $t=4$, (c) $t=5$, (d) $t=6$, and (e) $t=7$.

secondary vorticity entails the positive primary vortex and connects with the negative primary vortex. At this time, the primary vortex pair is about to pass the cylinder. The negative primary vortex is severely distorted, it almost reaches its minimum velocity $U_{x}$ and is pushed downwards into the $-y$ direction. After the vortex pair has passed the cylinder it re-attains its original shape as illustrated in Figures 16(d)-16(f).

The path lines of the vortex cores and the circular cylinder are plotted in Figure 17(b) for the time frame $t=10$ until $t=20$. Clearly, the cylinder moves on a circular path as it interacts with the passing vortex pair. The cylinder is first pushed downwards in the $-y$ direction by the approaching vortex pair. As the $U_{y}$ velocity plot in Figure 17(a) shows the cylinder begins to move upwards 


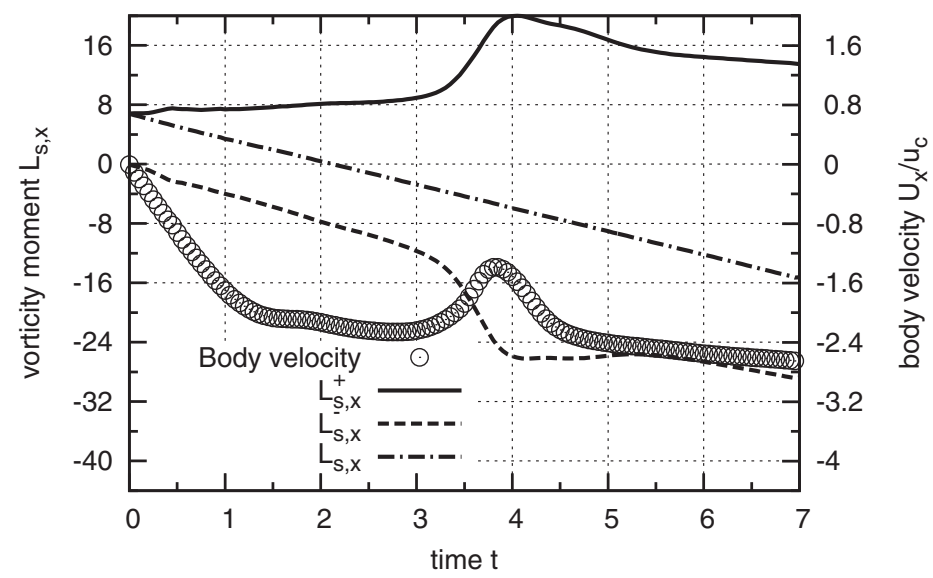

FIG. 15. Cylinder velocity and vorticity moment for the interaction of a vortex dipole with a neutrally buoyant cylinder subject to a constant non-dimensional body force $F_{x}=-0.0025 \pi D^{2}$ at $R e_{D}=500$.

towards its initial position shortly after time level $t=14$, i.e., after the vortex pair has passed the cylinder $y$ centerline as suggested by Figures 16(c) and 16(d). Furthermore, Figure 17(b) shows the path of the positive primary vortex to be much less disturbed by the presence of the cylinder than that of the negative primary vortex.

Figure 18(a) plots the different components of the conserved scalar $A_{z}$ as given by Eq. (31). It can be observed that the circular cylinder begins to rotate clockwise after time level $t=12$ making

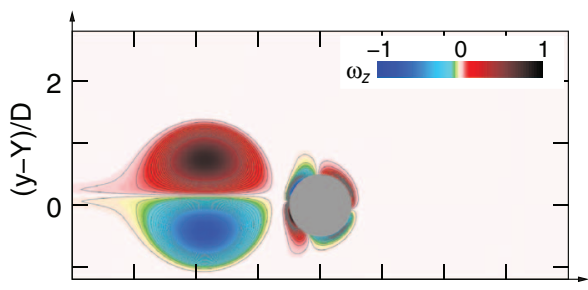

(a)

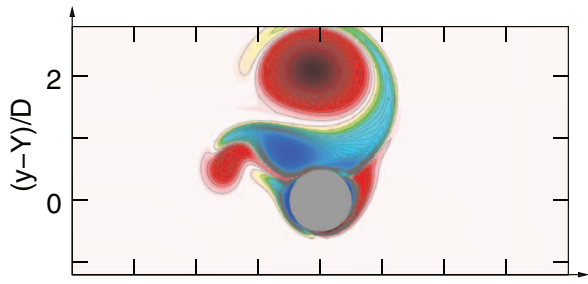

(c)

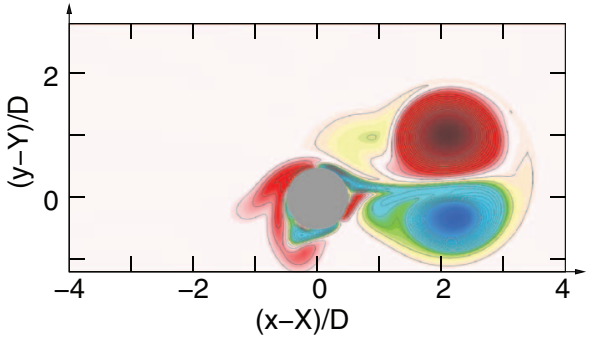

(e)

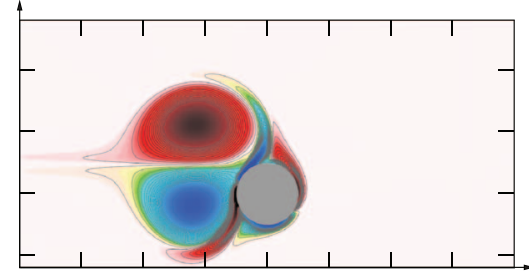

(b)

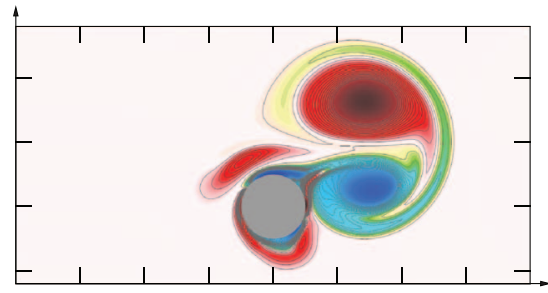

(d)

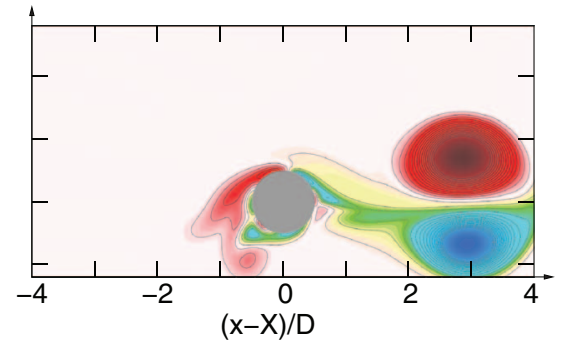

$(f)$

FIG. 16. Asymmetric interaction of a vortex dipole with a neutrally buoyant circular cylinder at $\operatorname{Re}_{D}=500$. Thirty vorticity contours in the range $-0.2 \leq \omega_{z} \leq 0.2$ are plotted in a moving frame of reference at (a) $t=10$, (b) $t=12$, (c) $t=14$, (d) $t=16$, (e) $t=18$, and (f) $t=20$. 


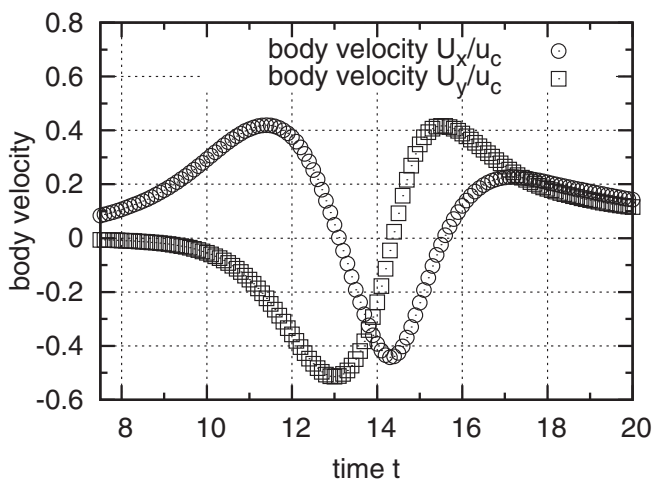

(a)

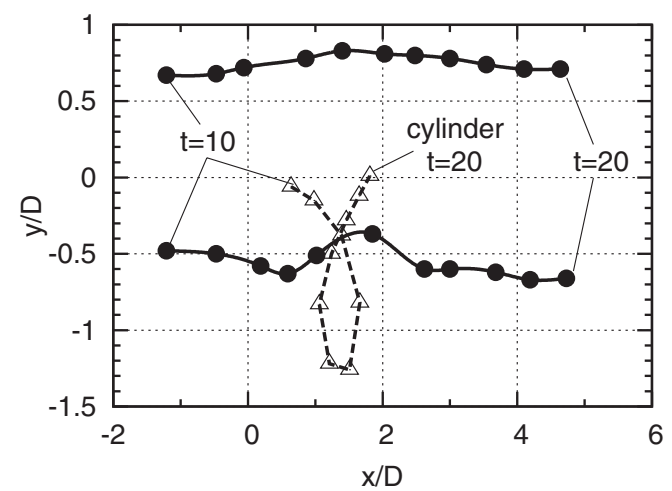

(b)

FIG. 17. Asymmetric interaction of a vortex pair with a neutrally buoyant circular cylinder: (a) normalized $x$ and $y$ body velocities and (b) path lines of the vortex cores $(\bullet)$ and the cylinder center of mass $(\triangle)$ for the asymmetric interaction. Data points are shown for each full time level in the range $10 \leq t \leq 20$.

the second term on the right-hand side of Eq. (31) non-zero. The contribution of this term to $A_{z}$, however, is relatively small compared to the other two terms as Figure 18(a) shows. Both of these contributions peak shortly after time level $t=14$, i.e., when the vortex pair has reached the cylinder $y$ center line, and nearly cancel out but for the rotation term. At time level $t=20$ after the vortex pair has passed the cylinder the first term and the third term on the right-hand side of (31) nearly vanish, while the cylinder continues to rotate.

Next, we show the correlation of the force and the time derivative of $\mathbf{L}_{s}$ for a multi-dimensional case. The asymmetric interaction of the vortex pair with a fixed circular cylinder is simulated. The vorticity field is illustrated in Figure 19 for different time levels showing similar features as the symmetric interaction shown in Figure 5. Likewise, the time history of the external force required to hold the cylinder in a fixed position illustrated in Figure 18(b) shows a similar behavior as in the symmetric fixed cylinder case. A significant difference between the symmetric and the asymmetric interaction is the non-zero lateral force on the cylinder in the asymmetric case represented by the force coefficient $C_{y, \text { ext }}$ in Figure 18(b). Both components of the external force coefficient vector show an excellent correlation with the time derivatives of the vorticity moment in the numerical simulation. This clearly verifies that Eq. (30) can be used to compute multi-dimensional body forces non-invasively based on a discrete and bounded measurement of the vorticity field.

Note that the computational confirmation of the correlation of the moment of force and the time derivative of $\mathbf{A}$ is difficult because of the very small moments involved in this test case. A clean

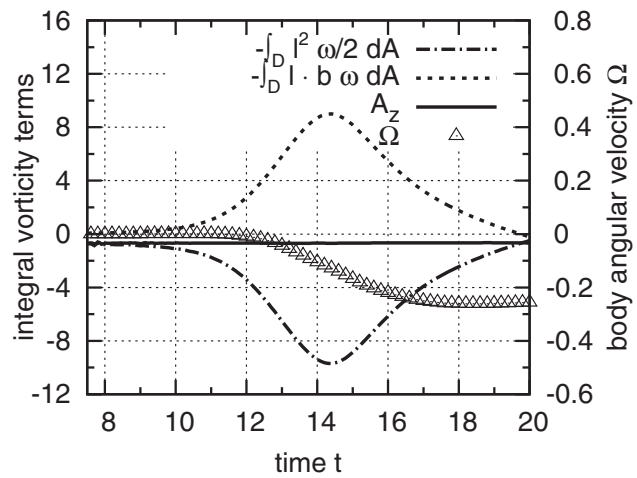

(a)

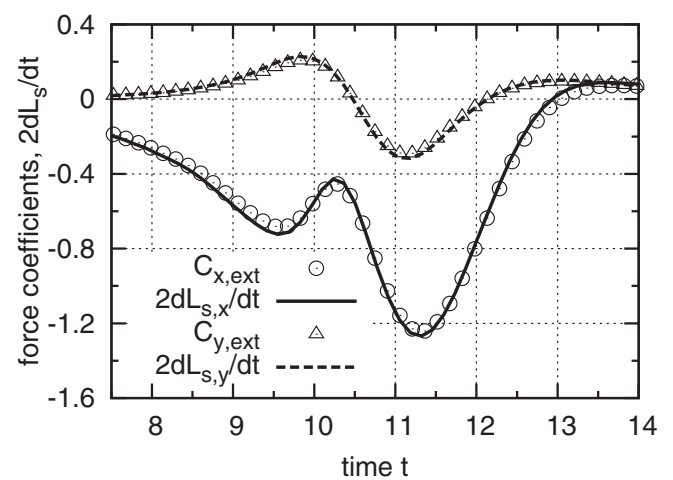

(b)

FIG. 18. Asymmetric interaction of a vortex pair with a circular cylinder: (a) body angular velocity $\Omega$ and the different terms contributing to the conserved scalar $A_{z}=-\frac{1}{2} \int_{D} l^{2} \omega d A-\int_{B} l^{2} \Omega d A-\int_{D} \mathbf{b} \cdot \mathbf{l} \omega d A$ for the neutrally buoyant cylinder case and (b) correlation of the external force coefficient $\mathbf{C}_{e x t}$ and the time variation $d \mathbf{L}_{s} / d t$ of the total vorticity moment. 


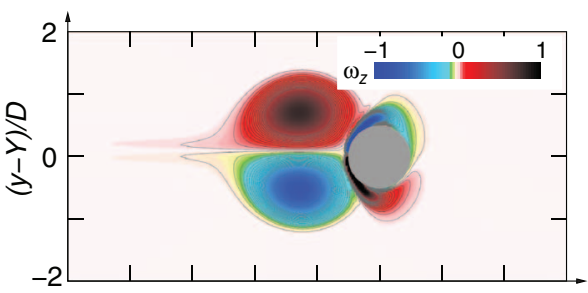

(a)

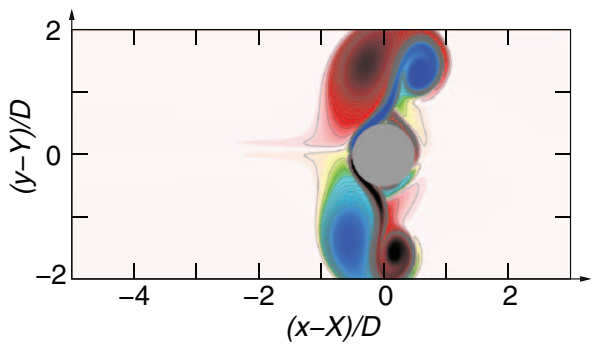

(c)

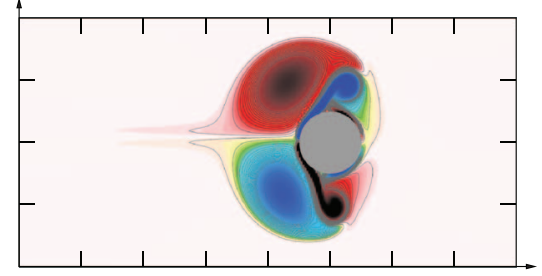

(b)

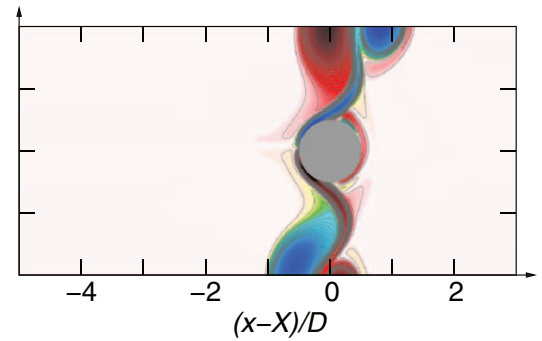

$(d)$

FIG. 19. Asymmetric interaction of a vortex dipole with a fixed circular cylinder. Thirty vorticity contours in the range $-0.2 \leq \omega_{z} \leq 0.2$ are plotted in a moving frame of reference at (a) $t=10$, (b) $t=11$, (c) $t=12$, and (d) $t=13$.

numerical confirmation would require a run of the test case at very small $R e$, which would, however, alter the vortex dynamics of the test case significantly. In the interest of a concise presentation, such an additional test case is omitted. Nevertheless, the conservation of $A_{z}$ evidenced in Figure 18(a) strongly suggests that a correlation between the moment of force and the time derivative can be obtained.

\section{SUMMARY}

A numerical study of the interaction of a vortex pair with a circular cylinder and novel correlations with momentum laws are presented in this paper. The expressions derived from the momentum laws allow the determination of the external forces on a body from the velocity field. This is verified in numerical simulations of vortex-pair-cylinder interactions, from which discrete and bounded data sets are obtained. Various symmetric and asymmetric cases for the interaction at different degree of complexity are considered ranging from a fixed body maintaining its position to a neutrally buoyant body accelerated by a constant thrust. The derived expressions show a reasonably small sensitivity with respect to the required resolution of the flow field, which is of significance for experimental investigations. In this respect, the presented relations are expected to be of particular interest for experiments involving living animals such as swimming fish, in which force measurements are nearly impossible.

Finally, the motion of the cylinder is related to the generation of secondary vorticity. It is shown that an increase of the secondary vorticity moment goes along with an acceleration of the cylinder and vice versa. The behavior of the cylinder motion after the initial acceleration suggests the occurrence of further vortex events, which are currently being investigated.

\section{ACKNOWLEDGMENTS}

This research was performed as part of the collaborative research center SFB 686, which is funded by the German Research Association (Deutsche Forschungsgemeinschaft (DFG)). The support is gratefully acknowledged. Banavara Shashikanth would like to acknowledge financial support in the form of a summer research fellowship from the Alexander von Humboldt Foundation. Daniel Hartmann is currently sponsored by the German Research Association (Deutsche Forschungsgemeinschaft (DFG)) under Grant No. HA 5535/2-1. The support is gratefully acknowledged. 
${ }^{1}$ H.-T. Ahn and Y. Kallinderis, "Strongly coupled flow/structure interactions with a geometrically conservative ALE scheme on general hybrid meshes," J. Comput. Phys. 219, 671-696 (2006).

${ }^{2}$ J. J. Allen, Y. Jouanne, and B. N. Shashikanth, "Vortex interaction with a moving sphere," J. Fluid Mech. 587, 337-346 (2007).

${ }^{3}$ G. K. Batchelor, An Introduction to Fluid Dynamics (Cambridge University Press, 2000).

${ }^{4}$ I. Borazjani, L. Ge, and F. Sotiropoulos, "Curvilinear immersed boundary method for simulating fluid structure interaction with complex 3D rigid bodies," J. Comput. Phys. 227, 7587-7620 (2008).

${ }^{5}$ I. Borazjani and F. Sotiropoulos, "On the role of form and kinematics on the hydrodynamics of self-propelled body/caudal fin swimming,” J. Exp. Biol. 213, 89-107 (2010).

${ }^{6}$ A.V. Borisov, I. S. Mamaev, and S. M. Ramodanov, "Motion of a circular cylinder and $n$ point vortices in a perfect fluid," Regular Chaotic Dyn. 8, 449-462 (2003).

${ }^{7}$ S. Childress, The Mechanics of Swimming and Flying (Cambridge University Press, 1981).

${ }^{8}$ J. O. Dabiri, S. P. Colin, J. H. Costello, and M. Gharib, "Flow patterns generated by oblate medusan jellyfish: Field measurements and laboratory analyses," J. Exp. Biol. 208, 1257-1265 (2005).

${ }^{9}$ I. Eames, M. Landeryou, and J. B. Flór, "Inviscid coupling between point symmetric bodies and singular distributions of vorticity," J. Fluid Mech. 589, 33-56 (2007).

${ }^{10}$ C. Farhat, K. van der Zee, and P. Geuzaine, "Provably second-order time-accurate loosely-coupled solution algorithms for transient nonlinear computational aeroelasticity," Comput. Methods Appl. Mech. Eng. 195, 1973-2001 (2006).

${ }^{11}$ H. Goldstein, Classical Mechanics (Addison-Wesley, 1980).

${ }^{12}$ D. Hartmann, M. Meinke, and W. Schröder, "An adaptive multilevel multigrid formulation for Cartesian hierarchical grid methods," Comput. Fluids 37, 1103-1125 (2008).

${ }^{13}$ D. Hartmann, M. Meinke, and W. Schröder, "A general formulation of boundary conditions on Cartesian cut cells for compressible viscous flow," AIAA Paper 2009-3878, 2009.

${ }^{14}$ D. Hartmann, M. Meinke, and W. Schröder, "An adaptive dual-mesh method for premixed combustion using the level set approach,” AIAA Paper 2010-1068, 2010.

${ }^{15}$ D. Hartmann, M. Meinke, and W. Schröder, "A level-set based adaptive-grid method for premixed combustion," Combust. Flame 158, 1318-1339 (2011).

${ }^{16}$ D. Hartmann, L. Schneiders, W. Schröder, and B. N. Shashikanth, "On the interaction of a vortex pair with a freely moving cylinder," AIAA Paper 2010-4749, 2010.

${ }^{17}$ D. Hartmann, W. Schröder, and S. Hemchandra, "Influence of hydrodynamic instability on the heat release transfer function of premixed flames," ASME Paper No. GT2010-22848, 2010.

${ }^{18}$ D. Hartmann, M. Meinke, and W. Schröder, "A strictly conservative Cartesian cut-cell method for compressible viscous flows on adaptive grids," Comput. Methods Appl. Mech. Eng. 200, 1038-1052 (2011).

${ }^{19}$ J. Homa, M. Lucas, and D. Rockwell, "Interaction of impulsively generated vortex pairs with bodies," J. Fluid Mech. 197, 571-594 (1988).

${ }^{20} \mathrm{M}$. S. Howe, "On the force and moment on a body in an incompressible fluid, with application to rigid bodies and bubbles at high and low Reynolds numbers," Q. J. Mech. Appl. Math. 48, 401-426 (1995).

${ }^{21}$ M. S. Howe, Acoustics of Fluid-Structure Interactions (Cambridge University Press, 1998).

${ }^{22}$ S. Kern and P. Koumoutsakos, "Simulations of optimized anguilliform swimming," J. Exp. Biol. 209, 4841-4857 (2006).

${ }^{23}$ H. Lamb, Hydrodynamics (Dover, 1932).

${ }^{24}$ B. van Leer, "Towards the ultimate conservative difference scheme. V. A second-order sequel to Godunov's method," J. Comput. Phys. 32, 101-136 (1979).

${ }^{25}$ J.-C. Lin and D. Rockwell, "Force identification by vorticity fields: Techniques based on flow imaging," J. Fluids Struct. 10, 663-668 (1996).

${ }^{26}$ M.-S. Liou and C. J. Steffen, Jr., “A new flux splitting scheme,” J. Comput. Phys. 107, 23-39 (1993).

${ }^{27}$ L. M. Milne-Thomson, Theoretical Hydrodynamics (Dover, 1996).

${ }^{28}$ U. K. Müller, B. L. E. van den Heuvel, E. J. Stamhuis, and J. J. Videler, "Fish foot prints: morphology and energetics of the wake behind a continuously swimming mullet (Chelon labrosus Risso)," J. Exp. Biol. 200, 2893-2906 (1997).

${ }^{29}$ J. C. Nauen and G. V. Launder, "Hydrodynamics of caudal fin locomotion by chub mackarel, scomber japonicus (scombridae)," J. Exp. Biol. 205, 1709-1724 (2002).

${ }^{30}$ F. Noca, D. Shiels, and D. Jeon, "A comparison of methods for evaluating time-dependent fluid dynamic forces on bodies, using only velocity fields and their derivatives," J. Fluids Struct. 13, 551-578 (1999).

${ }^{31}$ P. Orlandi, "Vortex dipoles impinging on circular cylinders," Phys. Fluids A 5(9), 2196-2206 (1993).

${ }^{32} \mathrm{H}$. Poincaré, Théorie des tourbillons. Leçons professées pendant le deuxième semestre 1891-1892 (G. Carre, Paris, 1893), see http://digital.library.cornell.edu/cgi/t/text/text-idx?c=math;idno=01800002.

${ }^{33}$ L. Quartapelle and M. Napolitano, "Force and moment in incompressible flows," AIAA J. 21, 911-913 (1983).

${ }^{34}$ S. M. Ramodanov, "Motion of a circular cylinder and $N$ point vortices in a perfect fluid," Regular Chaotic Dyn. 7, 291-298 (2002).

35 J. Roenby and H. Aref, “Chaos in body-vortex interactions,” Proc. R. Soc. London, Ser. A 466, 1871-1891 (2010).

${ }^{36}$ P. G. Saffman, Vortex Dynamics (Cambridge University Press, 1992).

${ }^{37}$ L. Schäfer, U. Dierksheide, M. Klaas, and W. Schröder, "Investigation of dissipation elements in a fully turbulent channel flow by tomographic particle-image velocimetry," Phys. Fluids 23, 035106 (2011).

${ }^{38}$ A. Schröder, R. Geisler, G. Elsinga, F. Scarano, and U. Dierksheide, "Investigation of a turbulent spot and a tripped turbulent boundary layer flow using time-resolved tomographic PIV,” Exp. Fluids 44, 305-316 (2008). 
${ }^{39}$ W. W. Schultz and P. W. Webb, "Power requirements of swimming: do new methods resolve old questions?," Integr. Comp. Biol. 42, 1018-1025 (2002).

${ }^{40} \mathrm{~B}$. N. Shashikanth, "Poisson brackets for the dynamically interacting system of a $2 \mathrm{D}$ rigid boundary and $n$ point vortices: The case of arbitrary smooth cylinder shapes," Regular Chaotic Dyn. 10, 1-14 (2005).

${ }^{41}$ B. N. Shashikanth, "Symmetric pairs of point vortices interacting with a neutrally buoyant 2D circular cylinder," Phys. Fluids 18, 127103 (2006).

${ }^{42}$ B. N. Shashikanth, J. E. Marsden, J. W. Burdick, and S. D. Kelly, "The Hamiltonian structure of a two-dimensional rigid cylinder interacting dynamically with $N$ point vortices," Phys. Fluids 14(3), 1214-1227 (2002).

${ }^{43}$ B. N. Shashikanth, A. Sheshmani, S. D. Kelly, and J. E. Marsden, "Hamiltonian structure for a neutrally buoyant rigid body interacting with $n$ vortex rings of arbitrary shape: The case of arbitrary smooth body shape," Theoret. Comput. Fluid Dyn. 22, 37-64 (2008).

${ }^{44}$ G. J. Sheard, T. Leweke, M. C. Thompson, and K. Hourigan, "Flow around an impulsively arrested circular cylinder," Phys. Fluids 19, 083601 (2007).

${ }^{45}$ B. Tao, J. Katz, and C. Meneveau, "Statistical geometry of subrid-scale stresses determined from holographic particle-image velocimetry measurements," J. Fluid Mech. 457, 35-78 (2002).

${ }^{46}$ E. D. Tytell, "Do trout swim better than eels? Challenges for estimating performance based on the wake of self-propelled bodies," Exp. Fluids 43, 701-712 (2007).

${ }^{47}$ E. D. Tytell and G. V. Lauder, “The hydrodynamics of eel swimming,” J. Exp. Biol. 207, 1825-1841 (2004).

${ }^{48}$ R. Verzicco, J. B. Flor, G. J. F. van Heijst, and P. Orlandi, "Numerical and experimental study of the interaction between a vortex dipole and a circular cylinder," Exp. Fluids 18, 153-163 (1995).

${ }^{49}$ C. H. K. Williamson and R. Govardhan, "Vortex-induced vibrations," Ann. Rev. Fluid Mech. 36, $413-455$ (2004).

${ }^{50}$ J. C. Wu, "Theory for aerodynamic force and moment in viscous flows," AIAA J. 19, 432-441 (1981).

${ }^{51}$ J.-Z. Wu and J.-C. Wu, "Interactions between a solid surface and a viscous compressible flow field," J. Fluid Mech. 254, 183-211 (1993). 